\title{
The power of randomness \\ in Bayesian optimal mechanism design ${ }^{\text {is }}$
}

\author{
Shuchi Chawla, David Malec*, Balasubramanian Sivan \\ Computer Sciences Department, University of Wisconsin-Madison, 1210 West Dayton Street, Madison, WI 53706
}

\begin{abstract}
We investigate the power of randomness in the context of a fundamental Bayesian optimal mechanism design problema single seller aims to maximize expected revenue by allocating multiple kinds of resources to "unit-demand" agents with preferences drawn from a known distribution. When the agents' preferences are single-dimensional Myerson's seminal work (1981) shows that randomness offers no benefit-the optimal mechanism is always deterministic. In the multi-dimensional case, when agents' preferences are arbitrarily correlated, Briest et al. (2010) showed that the gap between the expected revenue obtained by an optimal randomized mechanism and an optimal deterministic mechanism can be unbounded even when a single agent is offered only 4 services. We show that when the agents' values involve no correlation or a specific kind of positive correlation, the benefit of randomness is only a small constant factor.
\end{abstract}

Keywords: Mechanism design, Randomness, Revenue, Lotteries, Screening, Multi-product pricing JEL: D42, D44, D82

\section{Introduction}

A fundamental problem in mechanism design is to characterize the optimal selling strategy for a monopolist trying to maximize his revenue. One of the big successes in this area is Myerson's characterization (1981) of the optimal mechanism in settings where buyers' types are single-dimensional. The optimal mechanism can be described in simple terms: it is a maximizer of ironed virtual values, weakly monotone transformations of agents' values. Remarkably, the optimal mechanism is deterministic. For example, the optimal selling strategy for a single-good monopolist facing a single buyer is to offer the buyer a take-it-or-leave-it price that depends on the value distribution of the buyer. Unfortunately, as we move away from the single-parameter setting, the design of optimal mechanisms becomes far more complex. Manelli and Vincent (2007) noted that the class of all mechanisms that are optimal for some distribution of agent types includes nearly all mechanisms ${ }^{1}$; so no nontrivial characterization of optimal mechanisms is possible. In particular, this class includes mechanisms that use randomness. Thanassoulis (2004) gave specific examples of settings where randomized mechanisms are strictly better than deterministic ones. This begs the question: to what extent is randomness useful for revenue maximization? Are deterministic mechanisms near-optimal in multi-parameter settings as well?

In this paper we investigate the power of randomness in the context of the following archetypical multi-parameter optimal mechanism design problem-a single seller offers multiple kinds of service, and a number of "unit-demand" agents are each interested in buying any one of the services. Whereas in the settings considered by Myerson each agent has a single-dimensional type (namely a value for the item under sale), in our setting each agent has a multidimensional type characterized by a (different) value for each of the services offered by the seller. An example of this

\footnotetext{
This work was supported in part by NSF awards CCF-0830494 and CCF-0643763 and in part by a Sloan Foundation fellowship. A preliminary version of this paper appeared in Proceedings of the 11th ACM Conference on Electronic Commerce, ACM, 2010, under the same title.

${ }^{*}$ Corresponding author

Email addresses: shuchi@cs.wisc.edu (Shuchi Chawla), dmalec@cs.wisc.edu (David Malec), balu2901@cs.wisc.edu (Balasubramanian Sivan)

${ }^{1}$ This holds even if we restrict the type distributions to be independent across agents and items, the setting we consider in this paper.
} 

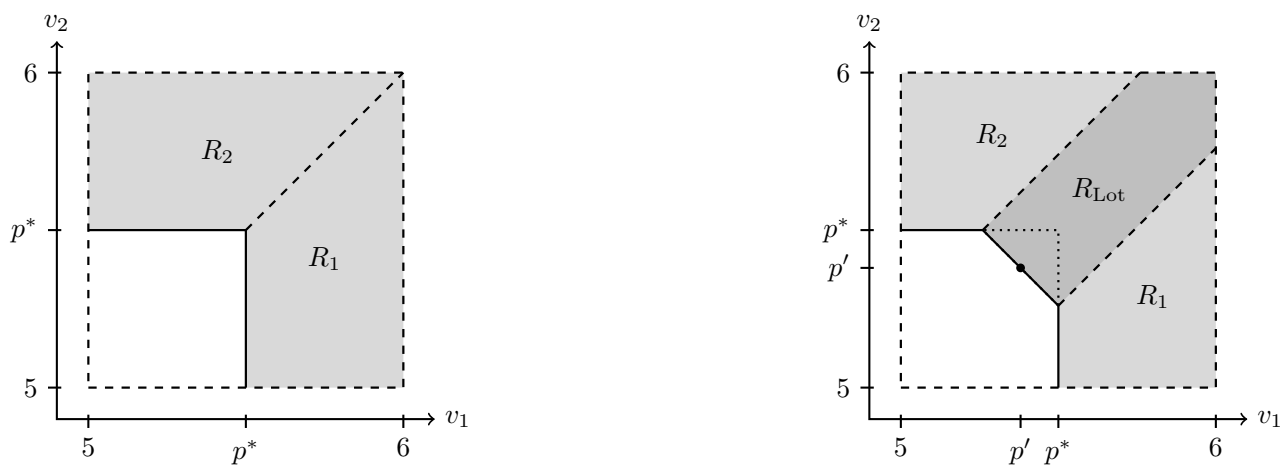

Figure 1: An example from Thanassoulis (2004) contrasting the optimal item and lottery pricings. The regions $R_{1}, R_{2}$, and $R_{\text {Lot }}$ denote the sets of valuations at which the agent buys item 1 , item 2 , and the $(1 / 2,1 / 2)$ lottery, respectively.

setting is the revenue maximization problem faced by an online travel agency selling airline tickets, hotel rooms, etc.; customers have different preferences over different available services, but are only interested in buying one. We study the Bayesian version of this problem: the distribution from which the buyers' preferences are drawn is known to the designer.

To answer these questions we must first understand the structure of randomized mechanisms in multi-dimensional settings. In the context of a single unit-demand agent and a seller offering multiple items, any deterministic mechanism is simply a price for each of the items with the agent picking the one that maximizes her utility (her value for the item minus its price). Likewise, randomized mechanisms can be thought of as pricings for distributions or convex combinations over items. These convex combinations are called lotteries. A risk-neutral buyer with a quasilinear utility function buys the lottery that maximizes his expected value minus the price of the lottery.

The following example due to Thanassoulis (2004) illustrates how lotteries work. Suppose that a seller offers two items for sale to a single buyer, and that the buyer's value for each of the items is independently and uniformly distributed in the interval $[5,6]$. The optimal deterministic mechanism for the seller is to simply price each of the items at $p^{*}=\$ 5.097$ (see Figure 1). In a randomized mechanism, the seller may in addition price a $(1 / 2,1 / 2)$ distribution over the two items at a slightly lower price of $p^{\prime}=\$ 5.057$. If the buyer purchases this lottery, the seller tosses a coin and allocates the first item to her with probability $1 / 2$ and the second with probability $1 / 2$. A buyer that is nearly indifferent between the two items would choose to buy the lottery because of its lower cost, rather than either one of the items. While the seller loses some revenue by selling the lower priced lottery with some probability, he gains by selling to a larger segment of the market (those that cannot afford either of the individual items but can afford the lower priced lottery). In this example the gain is more than the loss, so that introducing the lottery improves the seller's revenue.

Lotteries are thus a mechanism for sellers to screen buyers on their relative preferences for different items. For example, car dealerships often offer a discount on new cars when buyers do not have a strict preference for color. Likewise many travel agencies offer discounted vacation packages in which the vendor providing the service is unknown and depends on the demand at the time that the vacation is undertaken. This partitions the market into buyers that are indifferent among the different colors or vendors and buyers that greatly prefer some of the options over others.

In general, a randomized mechanism can offer to the buyer a menu of prices for arbitrarily many lotteries. We call such a menu a lottery pricing, and likewise a deterministic pricing an item pricing. In multiple-agent settings randomized mechanisms can be more complicated, but when viewed from the perspective of a single buyer they behave like a lottery pricing.

From the point of view of an optimizing designer, knowing the quantitative benefit from using lotteries can be crucial. On the one hand, optimizing revenue over the space of all lottery pricings is easier than optimizing over the space of all item pricings-Briest et al. (2010) show that the former can be done by solving a linear program. On the other hand, in general the optimal lottery pricing can contain as many different options on the menu as the number of different buyer types in the market. This may be infeasible to implement from a practical point of view. However, optimizing revenue over menus with few options (e.g., a single lottery in addition to item prices) appears to be harder 
than finding the optimal item pricing. If lotteries offer only a marginal improvement in revenue over item pricings, then the seller may be better off just using item prices.

Until recently, the largest gap known between item pricings and lottery pricings for a single agent was a gap of $3 / 2$ due to Pavlov (2006); for the special case where values for different items are independent, Thanassoulis (2004) gave the best gap example with a gap of 1.1. Briest et al. (2010) showed that in single-agent settings the gap between lottery pricings and item pricings can in fact be unbounded even with only 4 items. Specifically, they construct a discrete distribution over the agent's values, with each pair of value vectors having a large "angle" between them (and therefore representing different segments of the market). Then a lottery pricing, by offering different convex combinations over items to different segments, can obtain nearly the entire social value in the system, whereas a deterministic item pricing cannot price discriminate as effectively. Briest et al. show that when the number of items is at least 4 , an unlimited number of such value vectors can be packed into the distribution, leading to an unbounded gap between the revenues of the optimal item and lottery pricings. The value distributions they construct, however, are quite unnatural with the values of different items being correlated in a specific way.

In this paper we show that in single-agent problems with independent values (the setting considered by Thanassoulis) the revenue obtained by lottery pricings is no more than four times the revenue obtained by item pricings. We extend this result to settings involving multiple agents and general supply constraints, as well as a limited kind of correlation between values, again obtaining constant factor bounds on the benefit of randomness. While the constant factors we achieve are large in some cases, our results are in sharp contrast to the findings of Briest et al. where the improvement offered by lottery pricings is nearly as large as the number of different agent types in the market. Informally this implies that (when values are uncorrelated) randomized mechanisms cannot offer arbitrarily large improvements over deterministic ones. We believe that the factors we achieve can be improved considerably and the gap between randomized and deterministic mechanisms is much smaller in practice.

\section{Related work}

Randomness is a useful resource in mechanism design. In settings involving uncertainty, it allows the designer to hedge against adversarial input: in prior-free mechanism design where the designer has no information about buyers' values, (anonymous) deterministic mechanisms provably cannot obtain any guarantees on revenue and randomness is crucial (see, e.g., Hartline and Karlin, 2007, and references therein). Randomness is also useful when computation is a costly resource and the underlying optimization problem is computationally intractable (e.g. Dobzinski and Dughmi, 2009; Dughmi and Roughgarden, 2010). In the settings that we consider, neither of these effects are present: the designer knows the distribution from which agent types are drawn and we ignore computational issues. In our settings randomness helps for purely economic reasons - it gives the seller more latitude to price discriminate among buyers with different preferences.

Revenue-optimal mechanisms in multi-parameter settings are poorly understood. Following Myerson's characterization (1981) of optimal single-parameter mechanisms, there were a number of attempts to obtain simple characterizations of optimal mechanisms in the multi-parameter setting (McAfee and McMillan, 1988; Rochet and Chone, 1998; Manelli and Vincent, 2007), however no general-purpose characterization of such mechanisms is known (Manelli and Vincent, 2007). Chawla et al. (2010) gave the first approximations to optimal deterministic mechanisms for a large class of multi-parameter problems with unit-demand agents. This paper employs techniques developed in that work and one of the implications of our work is that the mechanisms developed there are approximately-optimal with respect to the optimal randomized mechanisms as well. Some of these results have been improved upon by Yan (2011) and Alaei (2011). Devanur et al. (2011) and Roughgarden et al. (2012) extend these approaches to give "prior-independent" multi-item auctions for unit-demand agents; these auctions give constant approximations to the (prior-dependent) revenue optimal auction.

Riley and Zeckhauser (1983) were the first to study the question of whether lotteries offer more revenue than item pricing; they showed that for a variety of single-parameter settings the optimal mechanism is deterministic. Subsequently Thanassoulis (2004) noted that there exist multi-parameter instances with valuations drawn from product distributions where randomness helps increase the revenue by about 8-10\%. Manelli and Vincent (2006) and Pavlov (2006) presented other examples with small gaps. Briest et al. (2010) were the first to uncover the extent of the benefit of randomization, as well as to study the hardness of finding the optimal randomized mechanism in multi-parameter settings. They showed that lottery pricings can be arbitrarily better than item pricings in terms of revenue even for the case of 4 items offered to a single agent. 
Our mechanism design setting with unit-demand agents is closely related to the standard setting for envy-free pricing problems considered in literature (Guruswami et al., 2005; Balcan and Blum, 2006; Balcan et al., 2008; Briest, 2006; Chawla et al., 2007); those works study the single-agent problem with a correlated value distribution and aim to approximate the optimal deterministic mechanism (item pricing). Our single-agent setting is most closely related to the work of Chawla et al. (2007) who gave a 3-approximation to the optimal deterministic mechanism for single-agent product-distribution instances, and builds upon techniques developed in that work. This factor was subsequently improved to a 2 in Chawla et al. (2010). Recently, a series of papers (Cai and Daskalakis, 2011; Daskalakis and Weinberg, 2012; Cai et al., 2012) presented a different approach for computationally finding arbitrarily good approximations to the optimal multi-item auction for agents with unit-demand or additive values.

\section{Our results and techniques}

We bound the gap between the optimal randomized revenue and the optimal deterministic revenue for multiparameter problems in a constructive fashion: given a good randomized mechanism we construct a deterministic one that is nearly as good in terms of revenue. In order to do so, we follow a technique introduced by Chawla et al. (2007), relating the optimal revenue for the multi-parameter setting to that for a certain single-parameter problem. Chawla et al. consider the optimization problem faced by a seller that wants to price $m$ items for a single unit-demand buyer with independent values for the items. They relate this setting to a hypothetical one in which the unit-demand buyer is replaced by $m$ "representatives" (henceforth reps) competing against each other, each interested in only one of the items. In the hypothetical setting the goal is to design a revenue-optimal mechanism that serves at most one of the reps at any instantiation of values. Chawla et al. argue that the increased competition among reps benefits the seller and leads to higher revenue. Formally, given an item pricing $\mathbf{p}$ there exists a truthful mechanism $A^{\mathbf{p}}$ that serves rep $i$ whenever $\mathbf{p}$ allocates item $i$ to the multi-parameter agent (that is, $A^{\mathbf{p}}$ has the "same" allocation rule as $\mathbf{p}$ ). Furthermore, the price that $A^{\mathbf{p}}$ charges is no less than the price that $\mathbf{p}$ charges for any instantiation of values. Therefore, the optimal deterministic revenue of the multi-parameter problem is bounded above by the optimal (deterministic or randomized) revenue, $S P$-OPT, for the corresponding single-parameter problem. Chawla et al. further show that any mechanism for the single-parameter problem with reps can be converted into a pricing for the original setting that gets at least a third of the revenue of the mechanism; this bound was subsequently improved to 2 in later work (Chawla et al., 2010). Thus, the converse of the above statement is also true (within a constant factor): SP-OPT is no more than twice the optimal deterministic revenue for the original problem. This suggests that bounding the revenue of the optimal lottery menu by SP-OPT would imply a small gap between lottery and item pricings.

Unfortunately, the upper bound via SP-OPT does not hold for lottery menus. Section 6 gives an example where SP-OPT for the single-parameter instance with reps is a factor of 1.13 smaller than that of the optimal lottery pricing for the multi-parameter instance. In fact, the mechanism $A^{\mathcal{L}}$ with the "same" allocation rule as a lottery pricing $\mathcal{L}$ may obtain zero revenue even when the lottery pricing obtains nonzero revenue. Nevertheless we show (Section 2) that the gap between SP-OPT and the optimal lottery pricing is no larger than a factor of 2 . Specifically, given a lottery pricing $\mathcal{L}$, we can construct two mechanisms, one being $A^{\mathcal{L}}$ and the other a Vickrey auction, such that the sum of the revenues of the two mechanisms is an upper bound on the revenue of the lottery pricing. Combining this with the result of Chawla et al. and an improvement over it in later work (Chawla et al., 2010), we get that for a single unit-demand agent multi-parameter problem, the gap between lottery pricings and item pricings is at most 4 .

Our result extends to multi-agent instances as well (Section 4). The simplest multi-agent setting we consider involves $n$ agents and $m$ items (with copies), where the seller faces a supply constraint for each of the items. A feasible allocation is a matching between agents and items that respects multiplicities of items. More generally, we consider settings where the seller faces a matroid feasibility constraint—any feasible allocation must be an independent set in a given matroid ${ }^{2}$ in addition to allocating at most one item per agent (see Section 4.3 for the definition of a matroid). In both of these cases we show that the gap between the expected revenues of the optimal randomized and the optimal deterministic mechanisms is a small constant factor. Once again our approach is to start with an optimal lottery pricing for the multi-parameter instance, construct an ensemble of mechanisms based on it for the corresponding single-parameter instance with reps, and then construct a so-called posted-pricing mechanism for the multi-parameter

\footnotetext{
${ }^{2} \mathrm{An}$ example of a matroid is the collection of all forests in a graph. The items in this case are the edges in a given graph; the seller can simultaneously sell any set of edges that does not contain a cycle.
} 
instance based on the mechanisms. The challenge is to ensure that the mechanisms we construct satisfy the nontrivial feasibility constraint.

In the most general setting that we consider, the theorem of Chawla et al. (2010) that we employ requires us to use a solution concept for deterministic mechanisms that is weaker than incentive compatibility, namely implementation in partial dominant strategy equilibrium (PDSE). They define an outcome to be in PDSE if every agent that has a dominant strategy follows it and other agents follow arbitrary undominated actions. Although this solution concept is weaker than incentive compatibility, it shares many of the good properties of IC: it is robust and predictable (to the extent that we care about, namely in terms of the revenue obtained).

Our factor-of- 4 gap for single-agent settings holds for instances where the values of the agent for different items are independent. For a unit-demand agent, this independence assumption is unrealistic. On the other end of the spectrum, however, Briest et al. show that with arbitrary correlations between item values the gap can be unbounded. We therefore examine the following natural model for values involving limited correlation, that we call the common base value model. The type of a unit-demand agent in this model is composed of $m+1$ independent components $\left(t_{0}, t_{1}, \cdots, t_{m}\right)$; the agent's value for item $i$ is $v_{i}=t_{0}+t_{i}$. Here $t_{0}$ can be thought of as the buyer's "base" value for obtaining any of the items, and the $t_{i}$ 's represent the buyer's perceived quality of the different items. The common base value introduces a positive correlation between values of different items. ${ }^{3}$

In the single-agent common base value setting we show that the gap between randomized and deterministic mechanisms is at most a factor of 8 . At a very high level, our approach is to partition the revenue of the optimal lottery pricing into what can be obtained though the base value of the agent alone, and what can be obtained if the base value is 0 . Formalizing this approach requires defining a new setting with $m+1$ items and an appropriate feasibility constraint over them that mirrors the original unit-demand one. In multi-agent settings, we need to devote extra care to ensure that the new feasibility constraint still belongs to the nice class of matroid intersections. This argument is presented in Section 5.

\section{Warm up: Single-agent setting}

In this section, we introduce our approach through the following most basic multi-parameter setting: we have a seller offering $n$ items for sale to a single unit-demand agent. That is, the agent is interested in buying at most one item. This model captures, for example, a buyer looking to buy a house or a TV set or a car. The agent has a value of $v_{i}$ for receiving item $i$, which is independent of all other values; $v_{i}$ is drawn from the (known) distribution $F_{i}$. The seller has no cost of production and wants to maximize his revenue from the sale.

Our goal is to study the gap between the revenue achievable from an optimal randomized truthful mechanism and that achievable from an optimal deterministic truthful mechanism. In this simple setting, deterministic truthful mechanisms are item pricings: the seller picks a vector of prices $\mathbf{p}$ for the items, and the buyer purchases the item $i$ that maximizes her utility, $v_{i}-p_{i}$, or buys nothing if all items provide negative utility.

On the other hand, randomized truthful mechanisms can be interpreted as lottery pricings that are defined as follows. An $n$-dimensional lottery is a vector $\ell=\left(q_{1}, \cdots, q_{n}, p\right)$ where $p$ is the price of the lottery and $\left(q_{1}, \cdots, q_{n}\right)$ is a probability distribution over the $n$ items, $\sum_{i \in[n]} q_{i} \leq 1$. A lottery pricing $\mathcal{L}=\left\{\ell_{1}, \ell_{2}, \cdots\right\}$ is a menu of (an arbitrary number of) lotteries offered by the seller to the buyer. As for item pricings, the buyer purchases the lottery that maximizes her utility. We assume that the agent is risk-neutral, and so her utility from a lottery $\ell=\left(q_{1}, \cdots, q_{n}, p\right)$ is given by $\sum_{i \in[n]} q_{i} v_{i}-p$.

Optimal item and lottery pricings are both difficult to understand and characterize. In order to relate them, we follow an approach developed by Chawla et al. (2007): we relate both to a third mechanism that we do understand well, namely the optimal mechanism for an appropriate single-parameter revenue maximization problem. The singleparameter problem we consider is a single-item $n$-agent auction where agent $i$ has a value $v_{i}$ for the item under sale. As before, $v_{i}$ is drawn independently from $F_{i}$. We call agent $i$ in this new setting a representative of the multi-parameter agent in the original setting (rep $i$ for short). Accordingly, we call an instance of the original problem $\mathcal{I}$ and the corresponding instance of the new single-item auction problem $\mathcal{I}^{\text {reps }}$.

\footnotetext{
${ }^{3}$ This model is similar to "multiplicative" value distributions that have been studied previously in the context of bundle pricing problems (see, e.g., Armstrong, 1999).
} 
Item pricings for $\mathcal{I}$ and deterministic mechanisms for $\mathcal{I}^{\text {reps }}$ are closely related, and in particular, their input-output behavior is identical. Both accept a value vector $\mathbf{v}$ drawn from the product distribution $\mathbf{F}=\prod_{i} F_{i}$ as input and return as output an index $i$ and a price to be paid. This observation allows us to map mechanisms from one setting to another (with an appropriate modification to the payment rule). It can be shown that the revenue of the optimal mechanism for $\mathcal{I}^{\text {reps }}$ is at most twice (but no smaller than) the revenue of the optimal item pricing for $\mathcal{I}$ (Chawla et al., 2007, 2010).

In order to prove that lottery pricings cannot be much better than item pricings, our goal is to show that the revenue of the optimal lottery pricing cannot be much larger than the revenue of the optimal mechanism for $\mathcal{I}^{\text {reps }}$. It is challenging to relate lottery pricings for $\mathcal{I}$ to (randomized) mechanisms for $\mathcal{I}^{\text {reps }}$. While it is possible to map the allocation rule of a lottery pricing to a mechanism in the latter setting, the main challenge is to apportion the price of a lottery across reps in such a way that a large fraction of the original payment is recovered and at the same time the mechanism is incentive compatible for the reps.

Before we present our main construction, we review Myerson's characterization (1981) of Bayesian incentive compatible mechanisms for single-parameter settings. Crucially we note that for Bayesian incentive compatibility, the payment rule induced by a monotone allocation rule is unique up to additive "shifts".

Theorem 1. (Myerson, 1981) A single-parameter mechanism with allocation rule $\mathbf{x}(\cdot)$ and payment rule $\mathbf{p}(\cdot)$ is Bayesian incentive compatible if and only if for all $i$ :

1. $x_{i}\left(v_{i}\right)$ is monotone nondecreasing in $v_{i}$ and

2. $p_{i}\left(v_{i}\right)=v_{i} x_{i}\left(v_{i}\right)-\int_{0}^{v_{i}} x_{i}(z) d z+p_{i}(0)$.

We now relate lottery pricings to mechanisms for $\mathcal{I}^{\text {reps }}$. Consider any lottery pricing $\mathcal{L}$. A natural candidate mechanism for $\mathcal{I}^{\text {reps }}$ based on $\mathcal{L}$ is one that implements the same allocation rule as $\mathcal{L}$ : at a value vector $\mathbf{v}$, if $\mathcal{L}$ allocates to the multi-parameter agent an item $i$ with probability $q_{i}$, then the candidate mechanism for $\mathcal{I}^{\text {reps }}$ allocates the item to rep $i$ with probability $q_{i}$. We note that this allocation rule is monotone nondecreasing in the values $v_{i}$ : if the multi-parameter agent increases his value for item $i$ keeping other values the same, then he may switch to a different lottery that allocates item $i$ with a higher probability, but never to one with a lower probability for item $i$. Therefore, by Theorem 1 there exists a truthful payment rule for this allocation rule in the $\mathcal{I}^{\text {reps }}$ setting. Call the resulting mechanism $\mathcal{A}^{\mathcal{L}}$. We normalize payments in this mechanism so that a rep with value 0 always pays 0 .

Ideally we would like to claim that the revenue of $\mathcal{A}^{\mathcal{L}}$ is close to that of $\mathcal{L}$, however this is not always the case. Let $n=2$ and consider a lottery pricing with a single lottery $\ell=(1 / 2,1 / 2,1)$. Whenever the agent has a value of 2 for any one of the items, he buys the lottery and pays 1 ; this holds in particular when the agent values both items at 2 . Now consider the corresponding mechanism $\mathcal{A}^{\mathcal{L}}$. Whenever any one of the reps values the item at 2 , both reps get the item with a $1 / 2$ probability each; in particular the other rep gets the item with a $1 / 2$ probability regardless of her report. So when both of the reps value the item at 2 , neither pays anything! Now if the values are $(2,2)$ with probability 1 , the lottery pricing $\mathcal{L}$ gets a revenue of 1 whereas $\mathcal{A}^{\mathcal{L}}$ gets a revenue of 0 . The point to note here is that this disparity in revenues arises only at value vectors where at least two reps have high enough values. But these are precisely the value vectors where another mechanism for $\mathcal{I}^{\text {reps }}$ obtains good revenue, namely the Vickrey auction. Therefore, we should be able to bound the loss in revenue from $\mathcal{A}^{\mathcal{L}}$ by the revenue of the Vickrey auction $\mathcal{V}$.

We now prove this formally. Let $\mathcal{R}^{M}(\mathbf{v})$ denote the revenue of a mechanism $M$ at value vector $\mathbf{v}$ and $\mathcal{R}_{i}^{M}(\mathbf{v})$ denote the contribution of rep $i$ to this revenue. Then we claim for all $\mathbf{v}$ :

$$
\mathcal{R}^{\mathcal{L}}(\mathbf{v}) \leq \mathcal{R}^{\mathcal{A}^{\mathcal{L}}}(\mathbf{v})+\mathcal{R}^{\mathcal{V}}(\mathbf{v}) .
$$

As an intermediate step towards this goal, it will help to first study a related mechanism $\tilde{\mathcal{A}}^{\mathcal{L}}$ that has the same allocation rule as $\mathcal{A}^{\mathcal{L}}$ but a different (shifted) pricing rule that follows the lottery pricing more closely. Given the lottery pricing $\mathcal{L}$, the mechanism $\tilde{\mathcal{A}}^{\mathcal{L}}$ forms a one-dimensional lottery pricing for each of the $n$ reps in $\mathcal{I}^{\text {reps }}$. Each rep then selects and is allocated her utility maximizing lottery. We denote the lottery pricing offered to rep $i$ as $\mathcal{L}_{i}$. For a given $\mathbf{v}_{-i}$ and $\ell=\left(q_{1}, q_{2}, \ldots, q_{n}, p\right) \in \mathcal{L}$, we add a lottery $\ell_{i}=\left(q^{\prime}, p^{\prime}\right)$ to $\mathcal{L}_{i}$, where

$$
\begin{aligned}
q^{\prime} & =q_{i} ; \text { and } \\
p^{\prime} & =p-\sum_{j \neq i} q_{j} v_{j} .
\end{aligned}
$$


Note that since $\tilde{\mathcal{A}}^{\mathcal{L}}$ offers each rep $i$ a menu of options that does not depend on that rep's reported value $v_{i}$, we may immediately conclude that $\tilde{\mathcal{A}}^{\mathcal{L}}$ is truthful. Since a rep always has the option to receive (and pay) nothing, we may also conclude that every rep always gets nonnegative utility from $\tilde{\mathcal{A}}^{\mathcal{L}}$ (i.e. it satisfies individual rationality). The following lemma shows that $\tilde{\mathcal{A}}^{\mathcal{L}}$ implements the same allocation rule as $\mathcal{L}\left(\right.$ or $\mathcal{A}^{\mathcal{L}}$ ).

Lemma 2. For any valuation vector $\mathbf{v}$, if the agent in $\mathcal{I}$ chooses $\ell$ from $\mathcal{L}$, then each rep $i$ in $\mathcal{I}^{\text {reps }}$ will select the $\ell_{i}$ corresponding to $\ell$ in $\tilde{\mathcal{A}}^{\mathcal{L}}$.

Proof: Fix some $\mathbf{v}$ and some $i$. Now, for any $\ell_{i} \in \mathcal{L}_{i}$, we can write the utility that rep $i$ receives from it as

$$
q^{\prime} v_{i}-p^{\prime}=q_{i} v_{i}-\left(p-\sum_{j \neq i} q_{j} v_{j}\right)=\sum_{j} q_{j} v_{j}-p,
$$

precisely the utility the original agent received from the lottery $\ell$ that $\ell_{i}$ was derived from. Since both the original agent and the rep are utility maximizers, the result follows.

We can now relate the revenue obtained by $\tilde{\mathcal{A}}^{\mathcal{L}}$ to that of $\mathcal{L}$.

Lemma 3. For any valuation vector $\mathbf{v}$ there exists an agent $i^{*}$ such that $\mathcal{R}^{\mathcal{L}}(\mathbf{v}) \leq \mathcal{R}_{i^{*}}^{\tilde{\mathcal{A}}^{\mathcal{L}}}(\mathbf{v})+\mathcal{R}^{\mathcal{V}}(\mathbf{v})$, where $\mathcal{V}$ denotes Vickrey's auction.

Proof: Fix a valuation vector $\mathbf{v}$. Let $i^{*}=\operatorname{argmax}_{i} v_{i}$. Let $\ell=\left(q_{1}, \ldots, q_{n}, p\right)$ be the lottery an agent with valuation vector $\mathbf{v}$ would select from $\mathcal{L}$ in $\mathcal{I}$. Then, $\mathcal{R}^{\mathcal{L}}(\mathbf{v})$ is precisely $p$, and so we can see that

$$
\mathcal{R}^{\mathcal{L}}(\mathbf{v})=\left(p-\sum_{i \neq i^{*}} q_{i} v_{i}\right)+\sum_{i \neq i^{*}} q_{i} v_{i}=\mathcal{R}_{i^{*}}^{\tilde{\mathcal{A}}^{\mathcal{L}}}(\mathbf{v})+\sum_{i \neq i^{*}} q_{i} v_{i} \leq \mathcal{R}_{i^{*}}^{\tilde{\mathcal{A}}^{\mathcal{L}}}(\mathbf{v})+\max _{i \neq i^{*}} v_{i},
$$

where the second equality follows from Lemma 2 and the definition of $\tilde{\mathcal{A}}^{\mathcal{L}}$, and the inequality follows from the fact that $\sum_{i} q_{i} \leq 1$ always. Since the revenue of Vickrey's auction for the setting $\mathcal{I}^{\text {reps }}$ on valuation vector $\mathbf{v}$ is precisely $\max _{i \neq i^{*}} v_{i}$, the claim follows.

Note that while the payment rule of $\tilde{\mathcal{A}}^{\mathcal{L}}$ makes it easy to relate its revenue from some agent to the total revenue of $\mathcal{L}$, we cannot conclude that $\mathcal{R}^{\mathcal{L}}$ is at most the total revenue of $\tilde{\mathcal{A}}^{\mathcal{L}}$ plus $\mathcal{R}^{\mathcal{V}}$, because $\tilde{\mathcal{A}}^{\mathcal{L}}$ may charge some reps negative prices, i.e. compensate them, to align their preferences with those of the multi-parameter agent. Instead we use the fact that $\tilde{\mathcal{A}}^{\mathcal{L}}$ and $\mathcal{A}^{\mathcal{L}}$ have identical allocation rules, and so have closely related payment rules.

In particular, Lemma 2 and Theorem 1 imply that the mechanisms $\tilde{\mathcal{A}}^{\mathcal{L}}$ and $\mathcal{A}^{\mathcal{L}}$ differ only by the payments they charge the reps at value 0 . Since $\tilde{\mathcal{A}}^{\mathcal{L}}$ is individually rational, it must charge a nonpositive payment to rep $i$ at $v_{i}=0$. Therefore, $\mathcal{A}^{\mathcal{L}}$ charges payments that are no smaller than the payments in $\tilde{\mathcal{A}}^{\mathcal{L}}$, and obtains more revenue than the latter. Noting that $\mathcal{A}^{\mathcal{L}}$ always charges nonnegative payments, we conclude the following.

Lemma 4. For any valuation vector $\mathbf{v}$ and rep $i$, we have that $\mathcal{R}_{i}^{\tilde{A}^{\mathcal{L}}}(\mathbf{v}) \leq \mathcal{R}_{i}^{\mathcal{A}^{\mathcal{L}}}(\mathbf{v}) \leq \mathcal{R}^{\mathcal{A}^{\mathcal{L}}}(\mathbf{v})$.

Lemmas 3 and 4 imply that $\mathcal{R}^{\mathcal{L}} \leq \mathcal{R}^{\mathcal{A}^{\mathcal{L}}}+\mathcal{R}^{\mathcal{V}} \leq 2 \mathcal{R}^{\mathcal{M}}$ where $\mathcal{M}$ is the optimal mechanism for $\mathcal{I}^{\text {reps }}$. We can combine this with a theorem of Chawla et al. (2010) (which is an improvement on a corresponding theorem of Chawla et al. (2007)) to get the main result of this section, namely that when values are distributed according to a product distribution, for any lottery pricing there exists an item pricing whose expected revenue is at least one fourth that of the lottery pricing.

Theorem 5 (Chawla et al. 2010, Theorem 8). Given a setting $\mathcal{I}$ where $\mathbf{F}$ is a product distribution, there exists an item pricing $\mathbf{p}$ for the setting $\mathcal{I}$ whose expected revenue is at least $1 / 2$ that of the optimal truthful mechanism for the setting $\mathcal{I}^{\text {reps }}$.

Theorem 6. Given a setting $\mathcal{I}$ where $\mathbf{F}$ is a product distribution, there exists an item pricing $\mathbf{p}$ for the setting $\mathcal{I}$ whose expected revenue is at least $1 / 4$ that of any lottery pricing for the setting $\mathcal{I}$. 


\section{Identically distributed values}

In the special case where the agent's values are identically and independently distributed across the items, the factor of four that we achieve above is improved to a factor of $\frac{2 e}{e-1} \approx 3.164$. The improvement comes from noting that for i.i.d. valuations, the factor of 2 in Theorem 5 can be improved to $\frac{e}{e-1}$ (Chawla et al., 2010).

\section{Pricings with a single lottery}

As we noted earlier, optimal lottery pricings can contain as many lotteries as the number of different buyer types in the market. In many practical settings, offering such large menus is unreasonable, and the seller may instead want to construct a menu with a single lottery in addition to item prices. A natural question is whether we can improve the bound in Theorem 6 by restricting our attention to lottery pricings with only one lottery. The following simple argument shows that the bound improves from a factor of 4 to a factor of just 2 .

For a lottery pricing of the given form, consider offering an agent either just the item pricings it contains, or just the single lottery it contains. Note that reducing the options in a lottery system only causes an agent to change their behavior if we remove their favorite option; thus, the combined revenue from offering these parts is at least the revenue of the original lottery pricing. Furthermore, an agent buying a lottery must value at least one of the items it randomizes over at the price of the lottery or higher. This implies that the revenue from offering a single lottery in isolation is no more than the revenue from offering each item in its support at the same price as the lottery. Since this gives two item pricings with combined revenue at least that of the original lottery pricing, one of the item pricings must give at least half of this amount.

\section{Multi-parameter mechanism design: Definitions and preliminaries}

In this section we will develop the notation and background that we need for the general multi-agent multiparameter revenue maximization problem.

\subsection{Bayesian multi-parameter unit-demand mechanism design}

We study the following Bayesian Multi-parameter Unit-demand (optimal) Mechanism Design (BMUMD) problem. There is one seller offering $m$ services indexed by the set $J=[m]$, and $n$ "unit-demand" agents indexed by the set $I=[n]$. Agents are risk-neutral and are each interested in buying any one of the $m$ services. Agent $i$ has value $v_{i j}$ for service $j$ which is a random variable. Values are drawn from the (publicly known) joint distribution $\mathbf{F}$. The seller faces no costs for providing service, but must satisfy certain feasibility constraints (e.g. supply constraints in a limited supply setting). We represent these feasibility constraints as a set system $\mathcal{S}$ over pairs $(i, j)$, that is, $\mathcal{S} \subseteq 2^{I \times J}$. Let $J_{i}=\{i\} \times J$. Each subset $A$ of $I \times J$ in $\mathcal{S}$ is a feasible allocation of services to agents, and $\left|A \cap J_{i}\right| \leq 1$ for all agents $i$. We use $\mathbf{v}_{-i}$ to denote the vector of values of all agents except agent $i$. The seller's goal is to maximize her revenue in expectation over the buyers' valuations.

The tuple $\mathcal{I}=(I \times J, \mathcal{S}, \mathbf{F})$ defines an instance of the BMUMD. A deterministic mechanism for this problem maps any set of bids $\mathbf{b}$ to an allocation $M(\mathbf{b}) \in \mathcal{S}$ and a pricing $\pi(\mathbf{b})$ with a price $\pi_{i}$ to be paid by agent $i$. A randomized mechanism maps a set of bids to a distribution over $\mathcal{S}$; we use $M(\mathbf{b})$ to denote this distribution.

We focus on the class of Bayesian incentive compatible (BIC) and (interim) individually rational (IR) mechanisms and will hereafter assume that $\mathbf{b}=\mathbf{v}$. We use $\mathcal{R}_{\mathcal{I}}^{M}(\mathbf{v})$ to denote the revenue of a mechanism $M$ for instance $\mathcal{I}$ at valuation vector $\mathbf{v}: \mathcal{R}_{\mathcal{I}}^{M}(\mathbf{v})=\sum_{i \in I} \pi_{i}(\mathbf{v})$ where $\pi$ is the pricing rule for $M$. We drop the subscript $\mathcal{I}$ when it is clear from the context. To aid disambiguation, we sometimes use $\mathcal{R}_{i}^{M}(\mathbf{v})$ to denote $\pi_{i}(\mathbf{v})$ for $M$. The expected revenue of a mechanism is $\mathcal{R}^{M}=\mathrm{E}_{\mathbf{v}}\left[\mathcal{R}^{M}(\mathbf{v})\right]$.

The feasibility constraint $\mathcal{S}$. We assume that the seller faces a matroid feasibility constraint. (See Section 4.2 for a definition of matroids.) In particular, there exists a matroid $\mathcal{S}^{\prime}$ over the groundset $I \times J$ such that each set $A$ belongs to $\mathcal{S}$ if and only if it is an independent set in $\mathcal{S}^{\prime}$ and satisfies $\left|A \cap J_{i}\right| \leq 1$ for all agents $i$. This captures multi-unit multi-item settings as a special case: here $J$ is a set of items with multiple copies each; A feasible allocation is any matching between items and agents that allocates no more than the available number of copies of each item. 
The distribution $\mathbf{F}$. In Section 4 we assume that each value $v_{i j}$ is distributed independently according to $F_{i j}$, that is, $\mathbf{F}=\prod_{i, j} F_{i j}$. In Section 5 we consider correlated values. In particular, each agent $i$ is assumed to have an $(m+1)$ dimensional type $\left\{t_{i 0}, \cdots, t_{i m}\right\}$ with each $t_{i j}$ being distributed independently according to a known distribution. Agent $i$ 's value for service $j$ is then given by $v_{i j}=t_{i 0}+t_{i j}$. Here we think of $t_{i 0}$ as being the agent's base value for receiving any service and the other $t_{i j}$ 's as representing the agent's different preferences for different services.

Single-parameter mechanism design. An instance of the Bayesian Single-parameter optimal Mechanism Design (BSMD) problem is given by the tuple $(I, \mathcal{S}, \mathbf{F})$. There is a seller offering $m=|I|$ services to $n=m$ singleparameter agents, with service $i$ targeted towards agent $i$. Agent $i$ 's value $v_{i}$ for getting served is a random variable. We use $\mathbf{v}_{-i}$ to denote the vector of values of all agents except agent $i$. The seller faces a feasibility constraint specified by a set system $\mathcal{S} \subseteq 2^{I}$, and is allowed to serve any set of agents in $\mathcal{S}$. As in the multi-parameter case, a mechanism $M$ for this problem is a function that maps a vector of values $\mathbf{v}$ to an allocation $M(\mathbf{v}) \in \mathcal{S}$ and a pricing $\pi(\mathbf{v})$. Myerson's seminal work describes the revenue maximizing mechanism for BSMD; this optimal mechanism is deterministic.

\subsection{Relating multi-parameter $M D$ to single-parameter $M D$}

In previous work we presented a general reduction from the multi-parameter optimal mechanism design problem to the single-parameter setting (Chawla et al., 2010). This approach begins with defining an instance $\mathcal{I}^{\text {reps }}$ of the BSMD given an instance $\mathcal{I}$ of the BMUMD. Our previous work then shows that for several kinds of feasibility constraints there exists a deterministic mechanism for $\mathcal{I}$ with revenue at least a constant fraction of that of the optimal mechanism for $\mathcal{I}^{\text {reps }}$. We state these results below without proof.

We begin by describing the instance $\mathcal{I}^{\text {reps }}$. Consider an instance $\mathcal{I}=(I \times J, \mathcal{S}, \mathbf{F})$ of the BMUMD. We define a new instance of the BSMD in the following manner. We split each agent in $\mathcal{I}$ into $|J|$ distinct representatives (hereafter called "reps"). Each rep $(i, j) \in I \times J$ is interested in only the service $(i, j)$ and behaves independently of (and potentially to the detriment of) other reps. The feasibility constraint on services stays the same as before. The instance is denoted $\mathcal{I}^{\text {reps }}$.

$\mathcal{I}^{\text {reps }}$ is similar to $\mathcal{I}$ except that it involves more competition (among different reps corresponding to the same multi-parameter agent). Therefore it is natural to expect that a seller can obtain more revenue in the instance $\mathcal{I}^{\text {reps }}$ than in $\mathcal{I}$. The following result shows that when the value distribution is a product distribution he cannot obtain too much more. In order to prove this result, Chawla et al. (2010) construct a mechanism for $\mathcal{I}$ based on the optimal mechanism for $\mathcal{I}^{\text {reps }}$. In some settings, the resulting mechanism does not admit a dominant strategy equilibrium. However, it obtains good revenue whenever any agent with a (weakly) dominant strategy follows that strategy (with other agents behaving arbitrarily). Formally, this is a notion of equilibrium that is weaker than dominant strategy equilibrium; Chawla et al. (2010) term it a partial dominant strategy equilibrium (PDSE). (See Section 5.)

Theorem 7 (Chawla et al. 2010, Theorems 7 and 11). Given an instance $\mathcal{I}$ of the BMUMD with unit-demand agents and a matroid feasibility constraint, there exists a deterministic mechanism for $\mathcal{I}$ that obtains an 8-approximation in PDSE to the revenue of any truthful mechanism for the instance $\mathcal{I}^{\text {reps }}$. In the special case where the set system consists of all valid matchings between agents and a multiset over items, there exists a truthful deterministic mechanism for $\mathcal{I}$ that obtains a 6.75-approximation to the revenue of any truthful mechanism for the instance $\mathcal{I}^{\text {reps }}$.

Given these bounds on the performance of the optimal deterministic mechanism for $\mathcal{I}$ in terms of the revenue of the optimal truthful (deterministic) mechanism for $\mathcal{I}^{\text {reps }}$, our goal through the rest of the paper is to relate the revenue of an optimal randomized mechanism for $\mathcal{I}$ to that of the optimal truthful(deterministic) mechanism for $\mathcal{I}^{\text {reps }}$. In Section 2, we accomplished this goal for the single-agent setting. In Section 4 we extend our argument to multi-agent settings with independent values. Section 5 deals with extensions to the common base value setting.

\section{Multi-agent settings}

We will now prove the main result of the paper, namely that the increase in a seller's revenue from using randomization in a multi-agent multi-parameter setting can be bounded by a small constant factor. We will extend the 
approach outlined in Section 2 for the special case of a single buyer: we bound the revenue of any BIC, IR randomized mechanism for an instance $\mathcal{I}$ of the BMUMD by those of three truthful deterministic mechanisms for the corresponding single-parameter instance with representatives, $\mathcal{I}^{\text {reps }}$.

Given a BIC, IR randomized mechanism $M$ for $\mathcal{I}$, we first study the properties of the mechanism $\mathcal{A}^{M}$ for $\mathcal{I}^{\text {reps }}$ that has the same allocation rule as $M$ (Section 4.1). Then, in an argument similar to the one in Section 2, we show that the revenue of $M$ can be bounded by the revenue of $\mathcal{A}^{M}$ plus the revenues of two VCG-style mechanisms for $\mathcal{I}^{\text {reps }}$ (Section 4.3). This argument requires us to use some properties of matroid set systems that we describe in Section 4.2.

\subsection{A mechanism for $\mathcal{I}^{\text {reps }}$}

Consider an instance $\mathcal{I}=(I \times J, \mathcal{S}, \mathbf{F})$ of the BMUMD. Given a randomized BIC, IR mechanism $M$ for $\mathcal{I}$, we define a mechanism $\mathcal{A}^{M}$ for the instance $\mathcal{I}^{\text {reps }}$ that also satisfies BIC and IR. As in the single-agent case, our goal is to relate the revenue of $M$ to the revenue of the mechanism $\mathcal{A}^{M}$ implementing the same allocation rule in $\mathcal{I}^{\text {reps }}$. To this end, we also need to make sure that $\mathcal{A}^{M}$ makes no positive transfers to the reps in $\mathcal{I}^{\text {reps }}$. Let $M(\mathbf{v})$ and $\pi(\mathbf{v})$ denote the allocation and payment rules, respectively, of the mechanism $M$. In an effort to unify our notation and theorem statements with Section 2, we define the quantities

$$
\begin{aligned}
q_{i j}\left(\mathbf{v}_{i}\right) & =\underset{\mathbf{v}_{-i}}{\mathrm{E}}\left[M_{i j}\left(\mathbf{v}_{-i}, \mathbf{v}_{i}\right)\right] ; \text { and } \\
p_{i}\left(\mathbf{v}_{i}\right) & =\underset{\mathbf{v}_{-i}}{\mathrm{E}}\left[\pi_{i}\left(\mathbf{v}_{-i}, \mathbf{v}_{i}\right)\right] .
\end{aligned}
$$

We are now ready to define the mechanism $\mathcal{A}^{M}$. For a given valuation vector $\mathbf{v}, \mathcal{A}^{M}$ will simulate the original mechanism $M$ on $\mathbf{v}$. It makes an allocation of $M_{i j}(\mathbf{v})$ to rep $(i, j)$, and charges a price of

$$
p_{i}\left(\mathbf{v}_{i}\right)-\sum_{k \neq j} q_{i k}\left(\mathbf{v}_{i}\right) v_{i k}+u_{i j}\left(\mathbf{v}_{i,-j}\right) .
$$

Note that this payment rule is similar to the one defined for mechanism $\tilde{\mathcal{A}}^{\mathcal{L}}$ in Section 2 except for the additive term $u_{i j}\left(\mathbf{v}_{i,-j}\right)$. The terms $u_{i j}\left(\mathbf{v}_{i,-j}\right)$ are normalization factors that ensure that $\mathcal{A}^{M}$ never makes positive transfers to the agents. In the proof of Lemma 8 below we describe how to set these terms so that the resulting mechanism is BIC, IR, and makes no positive transfers. For the sake of continuity we defer the proof of this lemma to the end of this subsection.

Lemma 8. If $M$ satisfies BIC and IR, then for appropriately defined $u_{i j}\left(\mathbf{v}_{i,-j}\right)^{\prime} s \mathcal{A}^{M}$ satisfies BIC and IR, and makes no positive transfers.

Following Section 2 as a guide, we would now like to upper bound the revenue of $M$ point-wise, i.e. for every value vector $\mathbf{v}$, by the revenue of $\mathcal{A}^{M}$ at $\mathbf{v}$ and a few other terms. Note, however, that we defined the payments of $\mathcal{A}^{M}$ not in terms of the payments $M$ actually charges, but in terms of their expectations (with respect to $\mathbf{v}_{-i}$ ). Thus, in order to achieve the sort of point-wise guarantee we want, we need to change how we account for the revenue of $M$. Specifically, we define the quantities

$$
\overline{\mathcal{R}}_{i}^{M}\left(\mathbf{v}_{i}\right)=\underset{\mathbf{v}_{-i}}{\mathrm{E}}\left[\mathcal{R}_{i}^{M}\left(\mathbf{v}_{-i}, \mathbf{v}_{i}\right)\right] ; \quad \text { and } \quad \overline{\mathcal{R}}^{M}(\mathbf{v})=\sum_{i \in I} \overline{\mathcal{R}}_{i}^{M}\left(\mathbf{v}_{i}\right) .
$$

Note that $\mathrm{E}_{\mathbf{v}}\left[\mathcal{R}^{M}(\mathbf{v})\right]=\mathrm{E}_{\mathbf{v}}\left[\overline{\mathcal{R}}^{M}(\mathbf{v})\right]$, and so it suffices to get a point-wise bound for $\overline{\mathcal{R}}^{M}(\mathbf{v})$. Furthermore, relating $\overline{\mathcal{R}}^{M}(\mathbf{v})$ to $\mathcal{R}^{\mathcal{A}^{M}}(\mathbf{v})$ is much more natural, since $\overline{\mathcal{R}}_{i}^{M}\left(\mathbf{v}_{i}\right)=p_{i}\left(\mathbf{v}_{i}\right)$.

While the mechanism $\mathcal{A}^{M}$ collects revenue from a total of $m n$ reps, we only focus on a small subset of these when establishing our bound. We denote this subset by $a(\mathbf{v})$. We choose a function $a(\mathbf{v})$ with the property that it includes at most one rep $(i, j)$ for any given $i$. Formally, a unit-demand function $a(\cdot)$ is a function mapping valuation vectors to sets of reps that respect the unit-demand constraint, i.e. for any valuation vector $\mathbf{v}$ and $i \in I,\left|a(\mathbf{v}) \cap J_{i}\right| \leq 1$.

The following generalization of Lemma 3 is our main characterization of $\mathcal{A}^{M}$. 
Lemma 9. For any unit-demand function $a(\cdot)$ and any valuation vector $\mathbf{v}$, we have

$$
\begin{aligned}
\overline{\mathcal{R}}^{M}(\mathbf{v}) & \leq \sum_{(i, j) \in a(\mathbf{v})} \mathcal{R}_{i j}^{\mathcal{A}^{M}}(\mathbf{v})+\sum_{(i, j) \notin a(\mathbf{v})} q_{i j}\left(\mathbf{v}_{i}\right) v_{i j} \\
& \leq \mathcal{R}^{\mathcal{A}^{M}}(\mathbf{v})+\sum_{(i, j) \notin a(\mathbf{v})} q_{i j}\left(\mathbf{v}_{i}\right) v_{i j} .
\end{aligned}
$$

Proof: We bound the contribution of each agent to the revenue term $\overline{\mathcal{R}}^{M}(\mathbf{v})$ independently. Fix some agent $i$, and let $\mathbf{q}_{i}\left(\mathbf{v}_{i}\right)$ and $p_{i}\left(\mathbf{v}_{i}\right)$ denote the expected allocation and payment for agent $i$ under mechanism $M$ (where the expectation is over the other agents' values $\mathbf{v}_{-i}$ ). Recalling the definition of $\mathcal{A}^{M}$, we can see that

$$
\begin{aligned}
\overline{\mathcal{R}}_{i}^{M}\left(\mathbf{v}_{i}\right)=p_{i}\left(\mathbf{v}_{i}\right) & =\left(p_{i}\left(\mathbf{v}_{i}\right)-\sum_{k \neq j} q_{i k}\left(\mathbf{v}_{i}\right) v_{i k}\right)+\sum_{k \neq j} q_{i k}\left(\mathbf{v}_{i}\right) v_{i k} \\
& \leq \mathcal{R}_{i j}^{\mathcal{A}^{M}}(\mathbf{v})+\sum_{k \neq j} q_{i k}\left(\mathbf{v}_{i}\right) v_{i k}
\end{aligned}
$$

for any $j$, where $\mathcal{R}_{i j}^{\mathcal{A}^{M}}(\mathbf{v})$ is the revenue of mechanism $\mathcal{A}^{M}$ from the rep $(i, j)$. The inequality follows from the fact that the normalization terms $u_{i j}\left(\mathbf{v}_{i,-j}\right)$ we chose are always nonnegative. Note that for bounding the portion of $\overline{\mathcal{R}}^{M}(\mathbf{v})$ corresponding to agent $i$, we have used the contribution of $\mathcal{A}^{M}$ obtained from just a single rep $(i, j)$. In particular, for a given agent $i$, we will pick the rep $(i, j) \in a(\mathbf{v})$ to bound $\overline{\mathcal{R}}_{i}^{M}\left(\mathbf{v}_{i}\right)$. Not every agent is guaranteed to have a rep in $a(\mathbf{v})$, however; for such an agent $i$, we instead use a formulation of the IR constraint for $i$ under $M$ : $p_{i}\left(\mathbf{v}_{i}\right) \leq \sum_{k} q_{i k}\left(\mathbf{v}_{i}\right) v_{i k}$. Adding the inequality (1) or the IR constraint (as appropriate) over all $i$, we get that for any unit-demand function $a(\cdot)$,

$$
\begin{aligned}
\overline{\mathcal{R}}^{M}(\mathbf{v}) & \leq \sum_{(i, j) \in a(\mathbf{v})} \mathcal{R}_{i j}^{\mathcal{A}^{M}}(\mathbf{v})+\sum_{(i, j) \notin a(\mathbf{v})} q_{i j}\left(\mathbf{v}_{i}\right) v_{i j} \\
& \leq \mathcal{R}^{\mathcal{A}^{M}}(\mathbf{v})+\sum_{(i, j) \notin a(\mathbf{v})} q_{i j}\left(\mathbf{v}_{i}\right) v_{i j},
\end{aligned}
$$

since we chose normalization terms $u_{i j}\left(\mathbf{v}_{i,-j}\right)$ in the payments of $\mathcal{A}^{M}$ so that the mechanism never made positive transfers to agents. This is precisely our claimed bound.

We now present the proof of Lemma 8.

Proof of Lemma 8: We first prove that $\mathcal{A}^{M}$ is BIC. Consider a rep $(i, j)$ with value $v_{i j}$. Our key observation is that the expected utility that the rep gets in $\mathcal{A}^{M}$ from reporting a value $v_{i j}^{\prime}$ can be related to the expected utility that the corresponding agent $i$ gets in $M$ from reporting a value vector $\left(v_{i j}^{\prime}, \mathbf{v}_{i,-j}\right)$ :

$$
\begin{aligned}
& \operatorname{Rep}(i, j) \text { 's expected utility from reporting } v_{i j}^{\prime} \text { in } \mathcal{A}^{M} \\
& =\underset{\mathbf{v}_{-i j}}{\mathrm{E}}\left[M_{i j}\left(\mathbf{v}_{-i j}, v_{i j}^{\prime}\right) v_{i j}-\left(p_{i}\left(\mathbf{v}_{i,-j}, v_{i j}^{\prime}\right)-\sum_{k \neq j} q_{i k}\left(\mathbf{v}_{i,-j}, v_{i j}^{\prime}\right) v_{i k}+u_{i j}\left(\mathbf{v}_{i,-j}\right)\right)\right] \\
& =\underset{\mathbf{v}_{i,-j}}{\mathrm{E}}[\underbrace{\sum_{k \in J} q_{i k}\left(\mathbf{v}_{i,-j}, v_{i j}^{\prime}\right) v_{i k}-p_{i}\left(\mathbf{v}_{i,-j}, v_{i j}^{\prime}\right)}_{\text {Agent } i \text { 's expected utility from reporting }\left(\mathbf{v}_{i,-j}, v_{i j}^{\prime}\right) \text { in } M}]-\underset{\mathbf{v}_{-i j}}{\mathrm{E}}\left[u_{i j}\left(\mathbf{v}_{i,-j}\right)\right]
\end{aligned}
$$

Note that the first term inside the expectation above is the utility that agent $i$ receives in $M$ from reporting $\left(\mathbf{v}_{i,-j}, v_{i j}^{\prime}\right)$; Since $M$ is BIC, for every $\mathbf{v}_{i,-j}$ this term is maximized when agent $i$ reports $\left(\mathbf{v}_{i,-j}, v_{i j}\right)$. The second term, on the other hand, is independent of $v_{i j}$. So the entire expression is maximized with $v_{i j}^{\prime}=v_{i j}$, and $\mathcal{A}^{M}$ is BIC.

We now proceed to show that $\mathcal{A}^{M}$ satisfies IR, that is, all reps get nonnegative expected utility. First, consider omitting the $u_{i j}\left(\mathbf{v}_{i,-j}\right)$ terms from the payments in $\mathcal{A}^{M}$. Then the equation above implies that rep $(i, j)$ gets expected utility from $\mathcal{A}^{M}$ that is exactly equal to the expected utility that agent $i$ gets from $M$ given $\mathbf{v}_{i}$. Thus, if the $u_{i j}\left(\mathbf{v}_{i,-j}\right)$ terms were all zero, then $\mathcal{A}^{M}$ would satisfy IR because $M$ satisfies IR. Note, however, that the $u_{i j}\left(\mathbf{v}_{i,-j}\right)$ terms cannot 
all be zero - an agent $i$ may value some item $j$ at $v_{i j}=0$, yet receive positive utility under $M$ via the allocation of some other item; the only way for $\mathcal{A}^{M}$ to match the utility of rep $(i, j)$ to that of agent $i$ is by making a positive transfer. To cancel this positive transfer, we choose

$$
u_{i j}\left(\mathbf{v}_{i,-j}\right)=\sum_{k \neq j} q_{i k}\left(\mathbf{v}_{i,-j}, 0\right) v_{i k}-p_{i}\left(\mathbf{v}_{i,-j}, 0\right),
$$

For a rep with value $v_{i j}=0$ this makes the payment as well as utility of the rep equal to 0 . To complete the proof we claim that (1) the rep gets positive expected utility at all $v_{i j}$, implying interim IR, and, (2) the rep makes nonnegative payments at all value vectors $\mathbf{v}$, implying no positive transfers. The first claim follows by noting that the mechanism is BIC and therefore the expected utility of any rep is a nondecreasing function of his value.

For the second claim, we use the fact that $M$ is BIC to note that for any rep $(i, j)$ and values $\mathbf{v}_{i,-j}$, the utility of the rep in expectation over $\mathbf{v}_{-i}$ is maximized when the rep reports his value truthfully. This is slightly stronger than the BIC condition proved above because it holds for all $\mathbf{v}_{i,-j}$ and not just in expectation over those values. This implies that the payment made by rep $(i, j)$ in expectation over $\mathbf{v}_{-i}$ is a nondecreasing function of his value $v_{i j}$. However, observe that rep $(i, j)$ 's payment is independent of $\mathbf{v}_{-i}$. Therefore, the ex post payment function is in fact a nondecreasing function of $v_{i j}$. Now, using the fact that payments are zero at $v_{i j}=0$, we conclude that payments are always nonnegative.

\subsection{Matroids and related set systems}

The set system $(X, \mathcal{S})$ over a universe $X$ with $\mathcal{S} \subseteq 2^{X}$ is called a matroid if it satisfies the following conditions:

1. (heredity) For every $A \in \mathcal{S}, B \subset A$ implies $B \in \mathcal{S}$.

2. (augmentation) For every $A, B \in \mathcal{S}$ with $|A|>|B|$, there exists $e \in A \backslash B$ such that $B \cup\{e\} \in \mathcal{S}$.

Sets in $\mathcal{S}$ are called independent, and maximal independent sets are called bases. A simple consequence of the above properties is that all bases are equal in size.

Here we give some examples of special matroid constraints. A $k$-uniform matroid on the universe $X$ is a matroid where every subset of $X$ of size at most $k$ is independent. A partition matroid $(X, \mathcal{S})$ is a union of two or more uniform matroids $\left\{\left(X_{i}, \mathcal{S}_{i}\right)\right\}_{i}$, where $\left\{X_{i}\right\}_{i}$ is a partition of $X$ and $\mathcal{S}=\left\{\cup_{i} A_{i}: A_{i} \subseteq \mathcal{S}_{i} \forall i\right\}$. A transversal matroid $(X, \mathcal{S})$ is defined by a bipartite graph $G=(V, E)$, where $\mathrm{V}$ is partitioned as $X \cup Y$ for some $Y$. The independent sets $\mathcal{S}$ are precisely those subsets of $X$ that can be matched one-to-one to a subset of $Y$ in $G{ }^{4}$ A set system $(X, \mathcal{S})$ is called a matroid intersection if there are two matroids $\left(X, \mathcal{S}_{1}\right)$ and $\left(X, \mathcal{S}_{2}\right)$, such that $\mathcal{S}=\mathcal{S}_{1} \cap \mathcal{S}_{2}$. An example of a matroid intersection is a matching in a bipartite graph.

The following proposition is a simple consequence of the above conditions on matroid set systems and will be useful in our analysis. We defer the proof to Appendix A.

Proposition 10. Let $B_{1}$ and $B_{2}$ be arbitrary independent sets in some matroid set system $\mathcal{S}$. Then there exists a set $B_{2}^{\prime} \subseteq B_{2}$ and a one to one function $g: B_{2}^{\prime} \rightarrow B_{1}$ such that for all $e \in B_{2}^{\prime}, B_{1} \backslash\{g(e)\} \cup\{e\}$ is independent in $\mathcal{S}$, and for all $e \in B_{2} \backslash B_{2}^{\prime}, B_{1} \cup\{e\}$ is independent in $\mathcal{S}$.

While we do not need the following proposition until Section 5, we present it now in the interest of collecting the results we need on matroids in one place. We present the proposition without proof (see, e.g. Korte and Hausmann, 1978, Theorems 1.7 and 3.2).

Proposition 11. Let $\mathcal{S}_{1}$ and $\mathcal{S}_{2}$ be arbitrary matroid set systems over a common ground set. Assume that each element $e$ in the ground set has a weight $w_{e}$. Then if we construct an independent set $\mathcal{G} \in \mathcal{S}_{1} \cap \mathcal{S}_{2}$ in a greedy fashion, i.e. by repeatedly selecting the highest weight element we can feasibly add to $\mathcal{G}$ until none are left, then

$$
\sum_{e \in \mathcal{G}} w_{e} \geq 1 / 2 \sum_{e \in S} w_{e}
$$

for any $S \in \mathcal{S}_{1} \cap \mathcal{S}_{2}$; furthermore, this holds no matter how we break ties (if any) when constructing $\mathcal{G}$.

\footnotetext{
${ }^{4}$ In fact, we can require that the subsets of $X$ in $\mathcal{S}$ have matchings to independent sets in some matroid $\left(Y, \mathcal{S}^{\prime}\right)$, and the result is still a transversal matroid. See, e.g., Aigner (1997) for more details.
} 


\subsection{Main theorem}

As noted earlier, the motivation for this setting arises in the context of multi-unit multi-item auctions. Consider, in particular, a seller with $m$ different items and $k_{j}$ copies of item $j$ for all $j$. Each of the $n$ unit-demand buyers have independently distributed values for each item. The seller's constraint is to allocate item $j$ to no more than $k_{j}$ agents, and to allocate at most one item to each agent. Note that the unit-demand constraint and the item supply constraints are each instances of partition matroids. Thus the system $\mathcal{S}$ in this setting is an intersection of two partition matroids.

More generally, in this section we consider set systems $\mathcal{S}$ that are intersections of the partition matroid corresponding to the unit-demand constraint over agents (call it $\mathcal{U}$ ) and an arbitrary other matroid over $I \times J$ (call it $\mathfrak{M}$ ).

As in the single-agent case, we want to bound the revenue of a randomized mechanism $M$ for $\mathcal{I}$ by the revenues of $\mathcal{A}^{M}$ and a suitably defined Vickrey auction. In order to do so, we can apply Lemma 9 to each value vector. The challenge is to bound the second term that we obtain in the lemma via a feasible mechanism for $\mathcal{I}^{\text {reps }}$. As in the singleagent case, we may bound the second term by the value of the second best item for each agent. However, this may be far larger than the value of any feasible allocation. For instance, if the seller has a single copy of some item $j$ and $j$ is every agent's highest valued item, then we cannot give every agent their highest valued item without violating the supply constraint. Instead we will use the fact that the $q_{i j}$ 's arise from distributions over feasible allocations, and the corresponding second term can therefore be bounded by revenue obtained from feasible allocations. We now present the details.

Lemma 12. Let $\mathcal{I}$ be an instance of the BMUMD. The revenue from any BIC, IR randomized mechanism $M$ for $\mathcal{I}$ is at most five times the expected revenue of the optimal mechanism for $\mathcal{I}^{\text {reps }}$.

Proof:

Consider any mechanism $M$ for $\mathcal{I}$ and recall the characterization of the corresponding mechanism $\mathcal{A}^{M}$ from Lemma 9:

$$
\overline{\mathcal{R}}^{M}(\mathbf{v}) \leq \mathcal{R}^{\mathcal{A}^{M}}(\mathbf{v})+\sum_{(i, j) \notin a(\mathbf{v})} q_{i j}\left(\mathbf{v}_{i}\right) v_{i j}
$$

where $a(\cdot)$ is any unit-demand function.

Recall that in the single-agent setting, we bound the second term in this characterization by the second highest value in $\mathbf{v}$. This essentially corresponds to taking $a(\mathbf{v})=\operatorname{argmax}_{i} v_{i}$. Likewise, here we will pick $a(\mathbf{v})$ to be the maximum value feasible set of reps; note that this is a unit-demand function. Let $A_{1}(\mathbf{v})$ denote this set (we drop the argument $\mathbf{v}$ wherever it is obvious). Summing Equation (2) over all value vectors we get

$$
\overline{\mathcal{R}}^{M}(\mathbf{v}) \leq \mathcal{R}^{\mathcal{A}^{M}}(\mathbf{v})+\underbrace{\sum_{(i, j) \notin A_{1}(\mathbf{v})} q_{i j}\left(\mathbf{v}_{i}\right) v_{i j}}_{T} .
$$

Our goal in the rest of the proof becomes to bound the second term above, labeled $T$. Informally, as in Section 2, we bound $T$ by the second best feasible set. To this end, we define the set $A_{2}$ as the maximum valued feasible set over the remaining $(I \times J) \backslash A_{1}$ reps:

$$
A_{2}(\mathbf{v})=\underset{S \in \mathcal{S} ; S \cap A_{1}(\mathbf{v})=\emptyset}{\operatorname{argmax}} v(S) .
$$

Note that our definition of $q_{i j}\left(\mathbf{v}_{i}\right)$ here does not exactly match up with the corresponding definition in Section 2. In that setting, the $q_{i}$ values represented overall expected allocations; here, they represent the allocation agent $i$ expects to receive knowing his or her own value, but not other agents' values. In particular, if an agent knows they have a relatively high value for a service with only small supply, it is reasonable for them to expect to receive it; but if every agent happens to value that service highly for a particular $\mathbf{v}$, most of the agents will be disappointed. The point is that for a particular value vector, the sum $T$ might give a value much larger than any feasible set, so we cannot hope to get a point-wise bound. However, $\mathbf{q}_{i}\left(\mathbf{v}_{i}\right)$ is, in fact, defined in terms of a feasible allocation, and so we can relate it to the values in $A_{2}$ in expectation. We formalize this in the following claim. 
Claim 1. The sum of values of all reps in $A_{2}$ is no less than $T$ in expectation:

$$
\underset{\mathbf{v}}{\mathrm{E}}\left[\sum_{(i, j) \in A_{2}(\mathbf{v})} v_{i j}\right] \geq \underset{\mathbf{v}}{\mathrm{E}}\left[\sum_{(i, j) \notin A_{1}(\mathbf{v})} q_{i j}\left(\mathbf{v}_{i}\right) v_{i j}\right] .
$$

Proof: This follows simply by computing

$$
\underset{\mathbf{v}}{\mathrm{E}}\left[\sum_{(i, j) \notin A_{1}(\mathbf{v})} q_{i j}\left(\mathbf{v}_{i}\right) v_{i j}\right]=\underset{\mathbf{v}}{\mathrm{E}}\left[\sum_{(i, j) \notin A_{1}(\mathbf{v})} M_{i j}(\mathbf{v}) v_{i j}\right] \leq \underset{\mathbf{v}}{\mathrm{E}}\left[\sum_{(i, j) \in A_{2}(\mathbf{v})} v_{i j}\right] ;
$$

the final inequality is a result of the fact that $M$ always allocates a feasible set, and that $A_{2}$ has the largest value among all feasible sets in the complement of $A_{1}$.

Next, we want to claim that a VCG-style mechanism can extract the value of the set $A_{2}$. At a high level, we can do so by constructing a mechanism that serves reps in $A_{1}$ and charges them prices that are at least as large as the values of the reps they displace in $A_{2}$. We need to ensure that each rep in $A_{2}$ becomes a price setter for at least one rep in $A_{1}$. If we faced either of the constraints $\mathcal{U}$ or $\mathfrak{M}$ alone, we could simply run a VCG mechanism and know that each displaced rep in $A_{2}$ would set the price for some rep in $A_{1}$. When we consider the intersection $\mathcal{U} \cap \mathfrak{M}$, however, it might be the case that a rep in $A_{2}$ is displaced by two different reps from $A_{1}$, each with respect to a different constraint, and so fails to set a price for either one (since neither can be assigned sole responsibility for the displacement). Our approach is to design two VCG-style mechanisms $M_{1}$ and $M_{2}$, which focus on extracting revenue related to displacements arising from the constraints $\mathcal{U}$ and $\mathfrak{M}$ respectively.

To formalize this, we use Proposition 10 to construct two maps from $A_{2}$ to $A_{1}$, one for each of the matroid constraints:

$$
\begin{aligned}
& g_{1}: A_{2} \rightarrow A_{1} \text { s.t. } \forall e \in A_{2}: \\
& g_{2}: A_{2} \rightarrow A_{1} \text { s.t. } \forall e \in A_{2}:
\end{aligned}
$$

$$
\begin{aligned}
& g_{1}(e) \text { is undefined and } A_{1} \cup\{e\} \in \mathcal{U} \text {, or } \\
& g_{1}(e) \text { is defined and } A_{1} \backslash\left\{g_{1}(e)\right\} \cup\{e\} \in \mathcal{U} \text {; and } \\
& g_{2}(e) \text { is undefined and } A_{1} \cup\{e\} \in \mathfrak{M} \text {, or } \\
& g_{2}(e) \text { is defined and } A_{1} \backslash\left\{g_{2}(e)\right\} \cup\{e\} \in \mathfrak{M} .
\end{aligned}
$$

It follows that for any $(i, j)$ in $A_{2}$, the set $A_{1} \cup\{(i, j)\} \backslash\left\{g_{1}(i, j), g_{2}(i, j)\right\}$ is a feasible set. Furthermore, by the optimality of $A_{1}, v_{i j} \leq v_{g_{1}(i, j)}+v_{g_{2}(i, j)}$. The maximality of $A_{1}$ implies that every element of $A_{2}$ has an image under either $g_{1}$ or $g_{2}$ or both.

We are now ready to define the mechanisms $M_{1}$ and $M_{2}$ by specifying their allocation rules. Given a valuation vector $\mathbf{v}$, the mechanism $M_{1}$ serves only those reps $(i, j)$ that belong to $A_{1}$ and for which $v_{i j} \geq v_{g_{1}^{-1}(i, j)} / 2$ (if $g_{1}^{-1}$ is defined at that point). Likewise, mechanism $M_{2}$ serves only those reps $(i, j) \in A_{1}$ that have $v_{i j} \geq v_{g_{2}^{-1}(i, j)} / 2$ (if defined). We note that $M_{1}$ and $M_{2}$ have monotone allocation rules, and are therefore IC. Truthful payments satisfying IR can be defined appropriately. Note that by our choice of allocation rule, whenever mechanism $M_{1}$ or $M_{2}$ serves rep $(i, j)$, it charges a payment of at least $v_{g_{1}^{-1}(i, j)} / 2$ or $v_{g_{2}^{-1}(i, j)} / 2$, respectively. Since both $M_{1}$ and $M_{2}$ serve subsets of $\mathcal{A}_{1}$, they are both feasible under $\mathcal{S}$ as well.

The following claim bounds from below the revenue for $M_{1}$ and $M_{2}$.

Claim 2. Twice the combined revenue of mechanisms $M_{1}$ and $M_{1}$ is no less than the sum of values of all reps in $A_{2}$ in expectation, i.e.,

$$
2\left(\mathcal{R}^{M_{1}}+\mathcal{R}^{M_{2}}\right) \geq \underset{\mathbf{v}}{\mathrm{E}}\left[\sum_{(i, j) \in A_{2}} v_{i j}\right] .
$$

Proof: Consider any rep $(i, j) \in A_{2}$, and the reps $g_{1}(i, j)$ and $g_{2}(i, j) \in A_{1}$ (if defined). Note that $A_{1}^{\prime}=A_{1} \cup(i, j) \backslash$ $\left\{g_{1}(i, j), g_{2}(i, j)\right\}$ is feasible. Suppose both $v_{g_{1}(i, j)}$ and $v_{g_{2}(i, j)}$ are less than $v_{i j} / 2$; then the set $A_{1}^{\prime}$ is a feasible set and $v\left(A_{1}^{\prime}\right)>v\left(A_{1}\right)$ which is a contradiction to the optimality of $A_{1}$. Thus one of $v_{g_{1}(i, j)}$ or $v_{g_{2}(i, j)}$ must be at least $v_{i j} / 2$ and so $M_{1}$ or $M_{2}$ charges that rep this amount, respectively. So we get that

$$
2\left(\mathcal{R}^{M_{1}}(\mathbf{v})+\mathcal{R}^{M_{2}}(\mathbf{v})\right) \geq \sum_{(i, j) \in A_{2}} v_{i j}
$$


for any $\mathbf{v}$; taking expectation over $\mathbf{v}$ yields the claim.

From equation (3) we can see that

$$
\mathcal{R}^{M}=\underset{\mathbf{v}}{\mathrm{E}}\left[\overline{\mathcal{R}}^{M}(\mathbf{v})\right] \leq \mathcal{R}^{\mathcal{A}^{M}}+\underset{\mathbf{v}}{\mathrm{E}}\left[\sum_{(i, j) \notin A_{1}(\mathbf{v})} q_{i j}\left(\mathbf{v}_{i}\right) v_{i j}\right]
$$

combining the above with Claims 1 and 2 completes the proof of Lemma 12.

Combining the lemma with Theorem 7 we obtain the following theorem.

Theorem 13. Given an instance $\mathcal{I}$ of the BMUMD with unit-demand agents and a matroid feasibility constraint, there exists a deterministic mechanism for $\mathcal{I}$ that obtains in PDSE at least a 1/40 fraction of the revenue of the optimal BIC, $I R$ randomized mechanism for $\mathcal{I}$. In the special case of multi-unit multi-item auctions, the revenue of any BIC, IR randomized mechanism is at most 33.75 times the revenue of the optimal deterministic mechanism for $\mathcal{I}$.

\section{Identically distributed values}

In the special case where for each agent, his values are identically and independently distributed across the items (but different agents' distributions need not be identical), the factor of 40 that we achieve above is improved to a factor of 15. The improvement comes from noting that for i.i.d. valuations, the factor of 8 in Theorem 7 can be improved to a factor of 3 (Chawla et al., 2010).

\section{Common base value correlation}

In the previous sections we considered settings where buyers have independent values for the different services offered. We now consider a more general model for agent types. Since the agents are unit-demand, we can think of the services being offered as perfect substitutes. A natural form of correlation, then, is for the agent to have some "base" value for being served (regardless of which service is received), plus an additive deviation specific to the particular service received.

Formally, in the common base value setting, agents' types consist of $(m+1)$ independently distributed values $\left\{t_{0}, t_{1}, \ldots, t_{m}\right\}$, with $t_{0}$ being the base value for getting served and $t_{i}$ being the additional benefit of obtaining service $i$; the agent's value for service $i$ becomes $v_{i}=t_{i}+t_{0}$. Henceforth, we use the abbreviation CBV to refer to the version of BMUMD with this type of common base value correlation.

\subsection{Warm up: single-agent setting}

Once again we introduce our techniques through the single-agent setting. At a high level, our approach is to "reduce" the CBV setting to BMUMD with independent values. In particular, given an instance $\mathcal{I}$ of the former, we construct an instance $\widehat{\mathcal{I}}$ of the latter such that a lottery pricing for the former can be converted into one for the latter without much loss in revenue, and conversely an item pricing for the latter can be converted into one for the former. Then we can just apply Lemma 12 to $\widehat{\mathcal{I}}$ to obtain a bound on the benefit of randomness for $\mathcal{I}$. The transformation from $\mathcal{I}$ to $\widehat{\mathcal{I}}$ is straightforward except that $\widehat{\mathcal{I}}$ does not satisfy the unit-demand constraint, and we need to modify the proof of Lemma 12 appropriately. We now present the details.

Theorem 14. Given an instance $\mathcal{I}$ of the $C B V$, there exists an item pricing $\mathbf{p}$ such that the revenue of any lottery menu $\mathcal{L}$ for $\mathcal{I}$ satisfies $\mathcal{R}^{\mathcal{L}} \leq 8 \mathcal{R}^{\mathrm{p}}$.

Proof: We begin by proving a bound with a weaker multiplicative factor of 9 and then show how to improve it to a factor of 8 . We first define an uncorrelated instance $\widehat{\mathcal{I}}$ of the BMUMD. $\widehat{\mathcal{I}}$ is a single-agent setting with $(m+1)$ items; we interpret the tuple $\left\{t_{0}, \ldots, t_{m}\right\}$ making up an agent's type in $\mathcal{I}$ as being the values of the agent in setting $\widehat{\mathcal{I}}$ for the $(m+1)$ items. In keeping with $\mathcal{I}$, the feasibility constraint we associate with $\widehat{\mathcal{I}}$ is that we may sell item 0 , and at most one additional item from among items $1, \ldots, m$. Note that the agent in $\widehat{\mathcal{I}}$ is not a unit-demand agent.

We first show how to convert a lottery menu $\mathcal{L}$ for $\mathcal{I}$ into a lottery menu $\widehat{\mathcal{L}}$ for $\widehat{\mathcal{I}}$ with no loss in revenue. For a lottery $\ell=\left(q_{1}, \ldots, q_{m}, p\right)$ in $\mathcal{L}$, we define $q_{0}=\sum_{i=1}^{m} q_{i}$, and add the lottery $\widehat{\ell}=\left(q_{0}, \ldots, q_{m}, p\right)$ to $\widehat{\mathcal{L}}$. Note that $\widehat{\ell}$ does not necessarily satisfy the requirement that the $q_{i}$ 's sum to at most one; it does, however, satisfy the feasibility 
constraint indicated for $\widehat{\mathcal{I}}$. Furthermore, fixing a type $t_{0}, \ldots, t_{m}$, the utility an agent in $\mathcal{I}$ receives from a lottery $\ell \in \mathcal{L}$ is

$$
\sum_{i=1}^{m} q_{i} v_{i}-p=\sum_{i=1}^{m} q_{i}\left(t_{i}+t_{0}\right)-p=\sum_{i=0}^{m} q_{i} t_{i}-p
$$

which is precisely the utility a corresponding agent in $\widehat{\mathcal{I}}$ would receive from the corresponding $\widehat{\ell} \in \widehat{\mathcal{L}}$. We thus have $\mathcal{R}^{\mathcal{L}}=\mathcal{R}^{\widehat{\mathcal{L}}}$.

Next we will prove an analog of Theorem 6 for $\widehat{\mathcal{I}}$. Note that we cannot apply that theorem directly because the instance does not satisfy the unit-demand constraint.

Consider the setting $\widehat{\mathcal{I}}^{\text {eps }}$ obtained by replacing the multi-parameter agent in $\widehat{\mathcal{I}}$ by $m+1$ single-parameter reps. Let $\widehat{\mathcal{M}}$ be the optimal mechanism for this instance. Likewise, let $\widehat{\mathcal{V}}$ be the Vickrey auction and $\mathcal{A}^{\widehat{\mathcal{L}}}$ be the mechanism with the same allocation rule as $\widehat{\mathcal{L}}$ for the instance $\widehat{\mathcal{I}}^{\text {reps }}$. Then, by following the proof of Lemma 3 , due to the less restrictive feasibility constraint $\left(\sum_{i=0}^{m} q_{i} \leq 2\right)$ we get

$$
\mathcal{R}^{\widehat{\mathcal{L}}} \leq \mathcal{R}^{\mathcal{A}^{\widehat{\mathcal{L}}}}+2 \mathcal{R}^{\widehat{\mathcal{V}}} \leq 3 \mathcal{R}^{\widehat{\mathcal{M}}}
$$

To complete the argument, we need to relate the revenue of the mechanism $\widehat{\mathcal{M}}$ for $\widehat{\mathcal{I}}^{\text {reps }}$ to that of a deterministic pricing for $\mathcal{I}$. To this end, a key observation is that our feasibility constraint for $\widehat{\mathcal{I}}^{\text {reps }}$ (carried over from $\widehat{\mathcal{I}}$ ) implies that $\widehat{\mathcal{M}}$ may make decisions about allocations and prices for rep 0 separately from those for reps $1, \ldots, m$; as such, $\widehat{\mathcal{M}}$ effectively consists of two mechanisms, one serving rep 0 and another serving reps $1, \ldots, m$, both under a unitdemand constraint. Now, the optimal mechanism for serving rep 0 is a pricing with a single price; applying this price to all of the items in $\mathcal{I}$ gives us a pricing with revenue at least as large as that of the optimal mechanism to serve rep 0 . For the optimal mechanism serving reps $1, \ldots, m$, Theorem 5 implies that there exists a pricing in $\widehat{\mathcal{I}}$ that obtains half the revenue of the mechanism; we apply the same pricing to $\mathcal{I}$ and note that the agent continues to select the same item under this pricing. Therefore, we can see that

$$
\mathcal{R}_{0}^{\widehat{\mathcal{M}}} \leq \mathcal{R}^{\mathbf{p}} \quad \text { and } \quad \mathcal{R}_{-0}^{\widehat{\mathcal{M}}} \leq 2 \mathcal{R}^{\mathbf{p}},
$$

where $\mathbf{p}$ is the optimal pricing for $\mathcal{I}, \mathcal{R}_{0}^{\widehat{\mathcal{M}}}$ is the revenue of $\widehat{\mathcal{M}}$ from rep 0 in $\widehat{\mathcal{I}}^{\text {reps }}$, and $\mathcal{R}_{-0}^{\widehat{\mathcal{M}}}$ is the revenue of $\widehat{\mathcal{M}}$ from the other reps in $\widehat{\mathcal{I}}^{\text {reps }}$. Putting everything together, we get

$$
\mathcal{R}^{\mathcal{L}} \leq 3 \mathcal{R}^{\widehat{\mathcal{M}}}=3\left(\mathcal{R}_{0}^{\widehat{\mathcal{M}}}+\mathcal{R}_{-0}^{\widehat{\mathcal{M}}}\right) \leq 3\left(\mathcal{R}^{\mathbf{p}}+2 \mathcal{R}^{\mathbf{p}}\right) \leq 9 \mathcal{R}^{\mathbf{p}} .
$$

In order to improve the factor from 9 to 8 , we employ better bounds on the contribution of $t_{0}$ and the contribution of other values to the revenue of a mechanism for $\widehat{\mathcal{I}}^{\text {reps }}$, depending on the type vector at which they are evaluated. Denote these quantities as $\mathcal{R}_{0}^{M}(\mathbf{t})$ and $\mathcal{R}_{-0}^{M}(\mathbf{t})$, respectively, for a mechanism $M$ at a particular valuation vector $\mathbf{t}$. As previously noted, the optimal mechanism $\mathcal{M}$ in $\widehat{\mathcal{I}}^{\text {reps }}$ treats rep 0 independently from reps $1, \ldots, m$; thus, we have that any mechanism $M$ in this setting must satisfy both $\mathcal{R}_{0}^{M} \leq \mathcal{R}_{0}^{\widehat{\mathcal{M}}}$ and $\mathcal{R}_{-0}^{M} \leq \mathcal{R}_{-0}^{\widehat{\mathcal{M}}}$.

Since we know that $\sum_{i=1}^{m} q_{i} \leq 1$, when $t_{0}$ is the maximum among all the $t_{i}$, Lemma 9 implies that

$$
\mathcal{R}^{\mathcal{L}}(\mathbf{t})=\mathcal{R}^{\widehat{\mathcal{L}}}(\mathbf{t}) \leq \mathcal{R}_{0}^{\mathcal{A}^{\widehat{\mathcal{L}}}}(\mathbf{t})+\mathcal{R}_{0}^{\widehat{\mathcal{V}}}(\mathbf{t})
$$

on the other hand, when one of $t_{1}, \ldots, t_{m}$ takes on the maximum value, we end up with, for some $i$,

$$
\mathcal{R}^{\mathcal{L}}(\mathbf{t})=\mathcal{R}^{\widehat{\mathcal{L}}}(\mathbf{t}) \leq \mathcal{R}_{i}^{\mathcal{A}^{\widehat{\mathcal{L}}}}(\mathbf{t})+2 \mathcal{R}_{i}^{\widehat{\mathcal{V}}}(\mathbf{t}) .
$$

Combining these two gives us a point-wise guarantee of

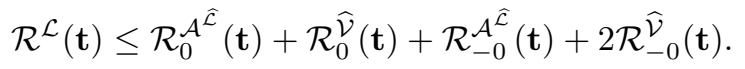

Therefore, if we let $\mathbf{p}$ be the optimal pricing for $\mathcal{I}$, we get

$$
\mathcal{R}^{\mathcal{L}} \leq \mathcal{R}_{0}^{\mathcal{A}^{\widehat{\mathcal{L}}}}+\mathcal{R}_{0}^{\widehat{\mathcal{V}}}+\mathcal{R}_{-0}^{\mathcal{A}^{\widehat{\mathcal{L}}}}+2 \mathcal{R}_{-0}^{\widehat{\mathcal{V}}} \leq 2 \mathcal{R}_{0}^{\widehat{\mathcal{M}}}+3 \mathcal{R}_{-0}^{\widehat{\mathcal{M}}} \leq 2 \mathcal{R}^{\mathbf{p}}+6 \mathcal{R}^{\mathbf{p}},
$$

implying the claimed bound of 8 . 


\subsection{Multi-agent settings}

We now consider the common base value model for multi-agent settings. As with the single-agent case, given an instance $\mathcal{I}=(I \times J, \mathcal{S}, \mathbf{F})$ of our problem, we construct a related setting $\widehat{\mathcal{I}}$ with independent values in such a way that any randomized mechanism $M$ for $\mathcal{I}$ corresponds naturally to a randomized mechanism $\widehat{M}$ for $\widehat{\mathcal{I}}$ that achieves the same expected revenue. Our argument has three main steps:

1. We define the related setting $\widehat{\mathcal{I}}$ and mechanism $\widehat{M}$.

2. We extend Lemma 12 to bound the revenue of the optimal mechanism for $\mathcal{I}$ in terms of the optimal mechanism for $\widehat{\mathcal{I}}^{\text {reps }}$.

3. We apply results of Chawla et al. (2010) to obtain good deterministic mechanisms for $\mathcal{I}$ that approximate the revenue of the optimal mechanism for $\widehat{\mathcal{I}}^{\text {reps }}$.

Step 1: Defining the setting $\widehat{\mathcal{I}}$ and mechanism $\widehat{M}$

The main idea behind our construction of the modified instance $\widehat{\mathcal{I}}$ remains unchanged from the single-agent case, namely, to separate the base value for service into its own service. We assign this extra service index 0 , and map an agent $i$ with values $\left(t_{i 0}+t_{i 1}, \ldots, t_{i 0}+t_{i m}\right)$ in $\mathcal{I}$ to an agent with values $\left(t_{i 0}, t_{i 1}, \ldots, t_{i m}\right)$ in $\widehat{\mathcal{I}}$. We construct the mechanism $\widehat{M}$ from $M$ by extending the allocation rule so that whenever $M$ allocates service $j$ to agent $i, \widehat{M}$ allocates both service $j$ and service 0 to agent $i$. We use the payment rule of $M$ without any changes for $\widehat{M}$. It is easy to see that $\widehat{M}$ is BIC and IR.

All that remains is to define the feasibility constraint $\widehat{\mathcal{S}}$ for the setting $\widehat{\mathcal{I}}$. In single-agent CBV settings, we used the constraint "service 0 plus at most one other service." In multi-agent CBV settings, we generalize this to "the projection of any feasible allocation for $\mathcal{I}$ onto service 0 , plus any feasible allocation for $\mathcal{I}$." To make this formal, write the feasibility constraint for $\mathcal{I}$ as $\mathcal{S}=\mathcal{U} \cap \mathfrak{M}$, where $\mathcal{U}$ represents the unit-demand constraint and $\mathfrak{M}$ is the matroid associated with the instance $\mathcal{I}$. Define constraint $\mathcal{S}_{0}$ on the reps for service 0 as

$$
\mathcal{S}_{0}=\left\{S \subseteq I \times\{0\}: \exists S^{\prime} \in \mathfrak{M} \text { such that }(i, 0) \in S \text { if and only if }(i, j) \in S^{\prime} \text { for some } j\right\} .
$$

Our overall feasibility constraint for the instance $\widehat{\mathcal{I}}$ is then the direct product $\widehat{\mathcal{S}}=\mathcal{S}_{0} \times \mathcal{S}$

The proposed feasibility constraint has several nice properties:

A. $\widehat{\mathcal{S}}$ is compatible with the modified mechanism $\widehat{M}$;

B. $\widehat{\mathcal{S}}$ is "close" to a unit-demand constraint (in particular, $\mathcal{S}_{0}$ and $\mathcal{S}$ are both unit-demand, and so every agent in $\widehat{\mathcal{I}}$ is allocated at most two services); and

C. $\widehat{\mathcal{S}}$ ensures that feasible allocations of service 0 and of services $1, \ldots, m$ are both related to feasible allocations in $\mathcal{S}$, but, importantly, allows decisions about these two to be made independently.

The second property above is what allows us to extend Lemma 12 to our current setting, and we discuss it further in the next subsection. The third property is critical to allowing us to find mechanisms for the original setting $\mathcal{I}$ that approximate the revenue of the optimal mechanism for $\widehat{\mathcal{I}}^{\text {reps; }}$; it means that a mechanism for $\widehat{\mathcal{S}}$ can be "split" into two mechanisms, one for service 0 and one for services $1, \ldots, m$, which respect $\mathcal{S}$ (or a projection of it).

Step 2: Bounding the optimal revenue for $\mathcal{I}$ via the optimal revenue for $\widehat{\mathcal{I}}^{\text {reps }}$

We now prove the following analogue to Lemma 12 for CBV instances. As in the proof of Theorem 14, we will use the fact that while the setting $\widehat{\mathcal{I}}$ is not unit-demand, it is "close" to unit-demand in that every feasible allocation can be covered by at most two unit-demand feasible allocations.

Lemma 15. Consider an instance $\mathcal{I}$ of the $C B V$. The revenue from any BIC, IR mechanism $M$ for $\mathcal{I}$ is at most nine times the expected revenue of the optimal mechanism for the instance $\widehat{\mathcal{I}}^{\text {reps }}$.

Proof: We follow the same framework as in the proof of Lemma 12; in fact, much of the proof goes through exactly as written there, and we refer the reader to that proof in such instances. 
In order to be able to leverage Lemma 9 in our proof, we need a unit-demand restriction of the feasibility constraint $\widehat{\mathcal{S}}$. We define $\widehat{\mathcal{S}}_{\mathcal{U}}=\mathcal{U} \cap\left(\mathcal{S}_{0} \times \mathfrak{M}\right)$ for this purpose. Note that $\widehat{\mathcal{S}}_{\mathcal{U}} \subseteq \widehat{\mathcal{S}}$, and further, for any $S \in \widehat{\mathcal{S}}$ there exist two sets $S_{1}, S_{2} \in \widehat{\mathcal{S}}_{\mathcal{U}}$ such that $S \subseteq S_{1} \cup S_{2}$.

We begin by considering the instance $\widehat{\mathcal{I}}=(I \times\{0\} \cup J, \widehat{\mathcal{S}}, \mathbf{F})$ and define, as before, a "best" set $A_{1}$ and a "second best" set $A_{2}$, and two VCG-style mechanisms $M_{1}$ and $M_{2}$. In this case, however, we define them with respect to the unit-demand feasibility constraint $\widehat{\mathcal{S}}_{\mathcal{U}}$ rather than the true feasibility constraint $\widehat{\mathcal{S}}$. We set

$$
A_{1}(\mathbf{v})=\underset{S \in \widehat{\mathcal{S}}_{\mathcal{U}}}{\operatorname{argmax}} v(S) \quad \text { and } \quad A_{2}(\mathbf{v})=\underset{S \in \widehat{\mathcal{S}}_{\mathfrak{U}} ; S \cap A_{1}(\mathbf{v})=\emptyset}{\operatorname{argmax}} v(S),
$$

respectively. Given a mechanism $M$ for $\mathcal{I}$, we can apply Lemma 9 to the mechanism $\widehat{M}$ for $\widehat{\mathcal{I}}$ as defined above and take an expectation over $\mathbf{v}$ to get

$$
\mathcal{R}^{M}=\mathcal{R}^{\widehat{M}} \leq \mathcal{R}^{\mathcal{A}^{\widehat{M}}}+\underset{\mathbf{v}}{\mathrm{E}}\left[\sum_{(i, j) \notin A_{1}(\mathbf{v})} q_{i j}\left(\mathbf{v}_{i}\right) v_{i j}\right]
$$

Recall that $\widehat{M}$ always allocates a set in $\widehat{\mathcal{S}}$, and that every such set can be covered by two sets in $\widehat{\mathcal{S}}_{\mathcal{U}}$. So, for any v, we have

$$
\sum_{(i, j) \notin A_{1}(\mathbf{v})} \widehat{M}_{i j}(\mathbf{v}) v_{i j} \leq 2 \sum_{(i, j) \in A_{2}(\mathbf{v})} v_{i j} .
$$

Then, we can apply the rest of the proof of Lemma 12 unchanged (in particular, Claim 2) with respect to the feasibility constraint $\widehat{\mathcal{S}}_{\mathcal{U}}$ to get that

$$
\mathcal{R}^{M} \leq \mathcal{R}^{\mathcal{A}^{\widehat{M}}}+2 \underset{\mathbf{v}}{\mathrm{E}}\left[\sum_{(i, j) \in A_{2}(\mathbf{v})} v_{i j}\right] \leq \mathcal{R}^{\mathcal{A}^{\widehat{M}}}+4\left(\mathcal{R}^{M_{1}}+\mathcal{R}^{M_{2}}\right)
$$

Since $\widehat{\mathcal{S}}_{\mathcal{U}} \subseteq \widehat{\mathcal{S}}$, all three of these mechanisms are feasible for the instance $\widehat{\mathcal{I}}^{\text {reps }}$, and the result follows.

\section{Step 3: Bounding the optimal revenue for $\widehat{\mathcal{I}}^{\text {reps }}$}

We now show that the revenue obtained by the optimal mechanism for $\widehat{\mathcal{I}}^{\text {reps }}$ is no more than a constant factor larger than that obtained by a deterministic mechanism for $\mathcal{I}$. Note that as in the single-agent case, the optimal mechanism for $\widehat{\mathcal{I}}^{\text {reps }}$ treats service 0 independently from services $1, \ldots, m$, and so we may bound the revenue from each of these separately. In the single-agent setting, it is straightforward to apply techniques of Chawla et al. (2010) to get approximately optimal pricings for the original setting $\mathcal{I}$. In the multi-agent setting, however, agents can affect each other's outcomes in nontrivial ways, and our approximation arguments necessarily become more complex. Dealing with these issues for service 0 and for services $1, \ldots, m$ is substantially different, and so we address these cases separately.

\section{Step 3a: Revenue from the base value}

Consider a mechanism $M$ for allocating service 0 for the instance $\widehat{\mathcal{I}}^{\text {reps }}$, that is, when the feasibility constraint is given by $\mathcal{S}_{0}$ alone. We can extend this mechanism to one for $\mathcal{I}$ as follows. For every agent that gets served at a certain price in $M$, we offer the agent any of the services 1 through $m$ at the same price. Then, there is some feasible allocation to the agents of services that obtains the same expected revenue as the mechanism $M$ for $\widehat{\mathcal{I}}^{\text {reps }}$. Of course the multiparameter agents in $\mathcal{I}$ may not choose to buy the services prescribed by this feasible allocation - each agent would instead buy the service with the most value. While an agent choosing to buy a different service contributes the same price to our revenue, it might block a very profitable sale to another agent who ends up not getting allocated at all. In order to show that we do not lose too much revenue in this manner, we employ a theorem of (Chawla et al., 2010) that states that we can start with an approximately-optimal mechanism $M$ that satisfies certain special properties, namely that it is a so-called posted price mechanism (PPM).

A PPM for an instance of the BMUMD uses the value distribution of the agents to come up with a price for each (agent, service) pair, and orders the pairs in decreasing order of price. It then offers services to agents at the respective 
prices in the aforementioned order subject to availability; agents make (binding) decisions to accept or reject the offers before the mechanism proceeds to the next pair in the ordering.

We note that the feasibility constraint $\mathcal{S}_{0}$ is a matroid. ${ }^{5}$ Therefore, we can employ the following theorem of Yan (2011), which improves upon a corresponding theorem of Chawla et al.

Theorem 16 (Yan 2011, Theorem 3.1 and Lemma 4.1). Given an instance of the BSMD with a matroid feasibility constraint, there exists a PPM with expected revenue at least a $(1-1 / e)$ fraction of that of the optimal mechanism for the instance.

We are now ready to bound the optimal revenue from service 0 for $\widehat{\mathcal{I}}^{\text {reps }}$.

Lemma 17. Given an instance $\mathcal{I}$ of the $C B V$, there exists a truthful deterministic mechanism for $\mathcal{I}$ whose revenue is at least a $1 / 2(1-1 / e)$ fraction of the revenue that an optimal mechanism for $\widehat{\mathcal{I}}^{\text {reps }}$ extracts from reps for service 0.

Proof: Consider the instance $\widehat{\mathcal{I}}^{\text {reps }}$ with just the constraint $\mathcal{S}_{0}$. We first apply Theorem 16 to obtain a PPM $P$ for this setting with a $(1-1 / e)$ fraction of the optimal expected revenue. We now construct a mechanism for $\mathcal{I}$ as follows. Consider agents in decreasing order of the prices at which $P$ offers service 0 to them in $\widehat{\mathcal{I}}^{\text {reps }}$. To each agent, offer any services that can be feasibly allocated at a uniform price equal to that in $P$, and allocate the service (if any) chosen by the agent.

Let us bound the revenue of this mechanism. Note that the revenue of $P$ is no more than the sum of prices corresponding to a maximum-total-price independent set in $\mathcal{S}_{0}$, or equivalently, a maximum-total-price independent set in $\mathcal{S}$. On the other hand, the constructed mechanism for $\mathcal{I}$ can be thought of as a greedy algorithm for $\mathcal{S}$, a matroid intersection constraint, that breaks ties in some arbitrary manner (namely according to agents' utilities). Proposition 11 then implies that this greedy algorithm obtains at least half of the revenue of the best independent set in $\mathcal{S}$. We therefore obtain the lemma.

Step 3b: Revenue from the service-specific values

Finally, we bound the revenue that the optimal mechanism for $\widehat{\mathcal{I}}^{\text {reps }}$ extracts from reps for services $1, \ldots, m$. At a high level, our approach is similar to that followed in the previous subsection to bound the revenue from reps for service 0 . We again invoke a theorem of Chawla et al. (2010) to get a posted price mechanism (PPM) for $\widehat{\mathcal{I}}$, and then analyze its performance when run in $\mathcal{I}$ in the natural way-offering services to agents in the same order and for the same prices.

Let us start by recalling the definition of posted price mechanisms for the BMUMD. A PPM for an instance of the BMUMD uses the value distribution of the agents to come up with a price for each (agent, service) pair, and orders the pairs in decreasing order of price. It then offers services to agents at the respective prices in the aforementioned order subject to availability; agents make (binding) decisions to accept or reject the offers before the mechanism proceeds to the next pair in the ordering. Agents know the prices that they are going to be offered for different services in the future, but not the availability of those services - that depends on which services get bought by other agents as a result of intervening offers. We remark that PPMs do not always induce a dominant strategy equilibrium. Instead Chawla et al. (2010) define a weaker solution concept called partial dominant strategy equilibrium (PDSE). An outcome is in PDSE if all agents that have dominant strategies follow said strategies and other agents follow undominated strategies. The results of Theorem 7 hold for PPMs implemented in PDSE.

Relating the outcome and revenue of a PPM applied to $\mathcal{I}$ and $\widehat{\mathcal{I}}$ presents two challenges: (1) agents in $\mathcal{I}$ may make different decisions from those in $\widehat{\mathcal{I}}$; (2) the offers made to any single agent are potentially interleaved by offers to other agents. The latter complicates an agent's decision to either accept the current offer, or wait for a future offer that brings in more utility but may get preempted by a sale to another agent. To deal with these challenges, we leverage a stronger revenue guarantee that Chawla et al. (2010) provide for PPMs for instances of the BMUMD. Let us define the favorite service of an agent to be the one that brings him the most utility, $j^{*}(i)=\operatorname{argmax}_{j}\left(v_{i j}-p_{i j}\right)$ for an agent $i{ }^{6}$ Note that

\footnotetext{
${ }^{5}$ Precisely, $\mathcal{S}_{0}$ can be seen to be a transversal matroid via a bipartite graph with $I \times\{0\}$ on one side and $I \times J$ on the other, and an edge between $(i, 0)$ and $(i, j)$ for every $i$ and $j$.

${ }^{6}$ If there are multiple utility-maximizing services, we break ties in favor of the one with the lowest price, i.e., in favor of latest offer made by the mechanism.
} 
when an agent is offered his favorite service, it is a dominant strategy for the agent to accept this service. In particular, the agent need not strategize about what offers he may receive in the future. The PPMs designed by Chawla et al. get a large expected revenue from agents who are only interested in buying their favorite service at the announced prices because the other services bring them negative utility. Focusing on this set of agents makes it easier for us to relate the agents' behavior in $\mathcal{I}$ and $\widehat{\mathcal{I}}$; in particular, each agent's favorite (utillity maximizing) service is the same in both settings.

The following stronger version of Theorem 7 is implicit from the proof in the cited work.

Theorem 18 (Chawla et al. 2010, Theorem 11). Given an instance $\widehat{\mathcal{I}}$ of the BMUMD with unit-demand agents and a matroid feasibility constraint, there exists a PPM P for $\widehat{\mathcal{I}}$, such that the expected revenue that it obtains in PDSE from agents that derive positive utility from only one service (by definition, their favorite one) at the given prices, is at least a $1 / 8$ fraction of the revenue of any truthful mechanism for the instance $\widehat{\mathcal{I}}^{\text {reps }}$.

We use the above theorem to approximate the revenue that the optimal mechanism for $\widehat{\mathcal{I}}^{\text {reps }}$ extracts from reps for services $1, \ldots, m$.

Lemma 19. Given an instance $\mathcal{I}$ of the $C B V$, there exists a deterministic mechanism for $\mathcal{I}$ achieving revenue in PDSE that is at least one sixteenth of that which the optimal mechanism for $\widehat{\mathcal{I}}^{\text {reps }}$ extracts from reps for services $1, \ldots, m$.

Proof: Consider the instance $\widehat{\mathcal{I}}^{\text {reps }}$ with just the services 1 through $m$ and the constraint $\mathcal{S}$. Applying Theorem 18 to the optimal mechanism for this instance, we get a PPM $P$ whose expected revenue over $\widehat{\mathcal{I}}$ from agents that only desire their favorite service is at least an eighth of the expected revenue that the optimal mechanism for $\widehat{\mathcal{I}}^{\text {reps }}$ extracts from reps for services $1, \ldots, m$. We apply the mechanism $P$ to setting $\mathcal{I}$ in the natural way: we offer services to agents in the same order and at the same prices as the ones used by $P$ for the setting $\widehat{\mathcal{I}}$.

We will now relate the revenue of $P$ from $\mathcal{I}$ to the revenue it obtains in $\widehat{\mathcal{I}}$ from agents that desire only their favorite service. Fix a type vector $\mathbf{t}$. Consider an agent $i$ that in mechanism $P$ in the setting $\widehat{\mathcal{I}}$ desires only his favorite service $j^{*}(i)$ at the given prices. Recall that $j^{*}(i)$ is still the agent's favorite service in the mechanism $P$ applied to setting $\mathcal{I}$. So, whenever the agent is offered $j^{*}(i)$, it is a dominant strategy for the agent to accept the service.

Formally, let $F(\mathbf{t})$ denote the set of (agent, service) pairs $(i, j)$ where $j$ is $i$ 's favorite service and the only service that the agent desires in the setting $\widehat{\mathcal{I}}$. Let $A(\mathbf{t})$ denote the set of (agent, service) pairs $(i, j)$ where $i$ accepts (purchases) $j$ in the setting $\mathcal{I}$. For any pair $(i, j)$ that belongs to $F(\mathbf{t})$ but not to $A(\mathbf{t})$ it must be the case that $P$ does not offer service $j$ to $i$ in $\mathcal{I}$ because it is blocked by a previous sale, i.e., it cannot be allocated feasibly. Accordingly, let $B(\mathbf{t})$ denote the set of (agent, service) pairs $(i, j)$ that are "blocked" in $\mathcal{I}$. Then we have $F(\mathbf{t}) \subseteq A(\mathbf{t}) \cup B(\mathbf{t})$.

Now we will focus on the set $A(\mathbf{t}) \cup B(\mathbf{t})$. Note that $P$ 's run on this set of (agent, service) pairs is essentially a greedy algorithm in which any pair that is not blocked is purchased. In particular, any pair that is not in $A(\mathbf{t}) \cup B(\mathbf{t})$ is offered but not accepted, and so does not effect $P$ 's run over this set of pairs. Since $\mathcal{S}$ is a matroid intersection constraint, we can apply Proposition 11 to conclude that the total revenue obtained by this run of the mechanism $P$ in $\mathcal{I}$, namely the sum of the prices corresponding to the pairs in $A(\mathbf{t})$, is at least half of the total price in any feasible subset of $A(\mathbf{t}) \cup B(\mathbf{t})$.

On the other hand, by the definition of $F(\mathbf{t})$, the expected revenue of $P$ in $\widehat{\mathcal{I}}$ from agents that only desire their favorite service is equal to the total price in $F(\mathbf{t})$. Since $F(\mathbf{t})$ is a feasible subset of $A(\mathbf{t}) \cup B(\mathbf{t})$, we may conclude that the revenue of $P$ from $\mathcal{I}$ when the type vector is $\mathbf{t}$ is at least half of the revenue that $P$ gets in $\widehat{\mathcal{I}}$ from agents desiring only their favorite service when the type vector is $\mathbf{t}$.

Taking expectations over $\mathbf{t}$ and applying Theorem 18 we get the lemma.

\section{Final approximation}

We now combine the lemmas from each of the above subsections to get the main theorem of this section.

Theorem 20. The revenue obtained by any BIC, IR randomized mechanism for an instance $\mathcal{I}$ of the CBV is at most $(162+18 /(e-1)) \approx 172.5$ times the revenue of the optimal IR deterministic mechanism for I implemented in partial dominant strategies. 
Proof: Consider any BIC, IR mechanism $M$ for $\mathcal{I}$. We apply Lemma 15 to $M$ to get that $\mathcal{R}_{\mathcal{I}}^{M} \leq 9 \mathcal{R}_{\mathcal{\mathcal { I }}_{\text {reps }}^{\mathcal{M}}}^{\mathcal{M} \text { where } \mathcal{M}}$ is the optimal mechanism for the setting $\widehat{\mathcal{I}}^{\text {reps }}$. Now, applying Lemmas 17 and 19 give us deterministic mechanisms for $\mathcal{I}$ that guarantee a $(e-1) / 2 e$ fraction and a $1 / 16$ fraction of the revenue that $\mathcal{M}$ extracts from reps for service 0 and services $1, \ldots, m$, respectively, in $\widehat{\mathcal{I}}^{\text {reps }}$. Thus, the better of these mechanisms guarantees revenue of at least $\mathcal{R}_{\widehat{\mathcal{I}}_{\text {reps }}^{\mathcal{M}}}^{\mathcal{M}} /(16+2 e /(e-1)) \geq \mathcal{R}_{\mathcal{I}}^{M} /(162+18 /(e-1))$.

\section{A gap example}

We showed in Sections 2 and 4 that the revenue of the optimal mechanism for $\mathcal{I}^{\text {reps }}$ gives an upper bound within constant factors to the revenue of an optimal randomized mechanism for $\mathcal{I}$. A natural question is whether it is possible to tighten our analysis to reduce the factor to 1, i.e., whether the optimal revenue for $\mathcal{I}^{\text {reps }}$ is a true upper bound on the revenue of an optimal randomized mechanism for $\mathcal{I}$. In this section we give a simple example where the revenue of a lottery pricing for a single-agent BMUMD instance $\mathcal{I}$ is strictly larger than the optimal revenue for the instance $\mathcal{I}^{\text {reps }}$; we then describe a generalization of this example where the former exceeds the latter by a factor of 1.13, the largest gap we know of.

The first instance we consider is defined as follows. There is a single agent with i.i.d. valuations for two items, each of which is drawn according to a discrete distribution with three point masses. Specifically, for $i=1,2$ we have that the valuation for item $i$ is distributed as

$$
v_{i} \sim \begin{cases}1 & \text { with probability } 1 / 2 ; \\ 2 & \text { with probability } 1 / 2-1 / H ; \text { and } \\ H & \text { with probability } 1 / H,\end{cases}
$$

where $H>2$ is a parameter to be fixed later.

Before we describe the optimal pricing and lottery, let us note some properties of this instance. Agents who value both items highly are so rare that their contribution to revenue will be negligible. Thus, we are concerned with extracting revenue from two groups of agents: those who place a low value on both items, and those who place a high value on exactly one item. Ideally, we would like to offer a low price to the former and a high price to the latter, but item pricings do not give us the ability to target agents in this way. In the setting with reps, we expect that increased competition leads to increased revenue. However, the rarity of the event that both reps have high values means that competition does little to improve things. On the other hand, lotteries are perfectly suited to this example since they allow us to screen agents based on the strength of their preferences between the two items. Essentially, randomization lets us add a "new item" to our offerings, which is really a $(1 / 2,1 / 2)$ chance on the two items. Agents with low values place a low value on this item; agents with exactly one high value place an intermediate value on this item. Thus, we can place a low price on the lottery to serve the former agents, and yet still sell items outright to the latter agents at an increased price. This improved market segmentation leads to the revenue increase that we shall shortly see.

Consider the optimal revenue in the setting $\mathcal{I}^{\text {reps }}$. If we relax the feasibility constraint so that we may serve both reps at once, we can only improve the optimal revenue. On the other hand, this relaxation means that allocation decisions for the two reps can be made independently, so the optimal mechanism just offers each rep a fixed price for service. Note, however, that by our choice of distribution, any price achieves expected revenue of at most 1 . Thus, we can upper bound the revenue of the optimal mechanism for the setting $\mathcal{I}^{\text {reps }}$ by 2 . The same argument also shows that the revenue from an optimal item pricing in the setting $\mathcal{I}$ is also upper bounded by 2 .

We now proceed to give a lottery system that achieves expected revenue strictly exceeding 2 . Consider the following lottery pricing $\mathcal{L}$ for $\mathcal{I}$ :

$$
\mathcal{L}=\left\{\left(\frac{1}{2}, \frac{1}{2}, \frac{3}{2}\right),\left(1,0, \frac{H+1}{2}\right),\left(0,1, \frac{H+1}{2}\right)\right\} .
$$

The first two coordinates in every lottery denote the probabilities with which items 1 and 2 are offered by that lottery and the third coordinate is the price. So this corresponds to putting a price of $(H+1) / 2$ on each item, as well as offering a lottery that allocates each item with probability $1 / 2$ at a price of $3 / 2$. Then we can see that if the agent values exactly one of the items at $H$, they will buy that item outright; if they value both items at 1 , they will buy 
nothing; and otherwise, they will buy the lottery. Thus, with probability $3 / 4$, the agent makes a purchase; and with probability $2 / H(1-1 / H)$ they choose to buy an item outright. The expected revenue will be

$$
\frac{3}{4} \cdot \frac{3}{2}+\left(\frac{H+1}{2}-\frac{3}{2}\right) \frac{2}{H}\left(1-\frac{1}{H}\right)=\frac{17}{8}-\frac{3}{H}+\frac{2}{H^{2}},
$$

which is strictly greater than 2 for $H=24$.

We now give a generalization of the above example to continuous distributions, and show that the gap in expected revenues increases to a factor of 1.13. The new instance $\mathcal{I}$ is defined as follows. There is still a single agent with i.i.d. valuations for two items; now the items' values are distributed according to a continuous equal-revenue distribution bounded at $H$. Formally, the valuations $v_{1}$ and $v_{2}$ for items 1 and 2 have distributions $F_{1}$ and $F_{2}$ such that

$$
F_{1}(x)=F_{2}(x)= \begin{cases}1-1 / x & 1 \leq x<H ; \text { and } \\ 1 & x=H .\end{cases}
$$

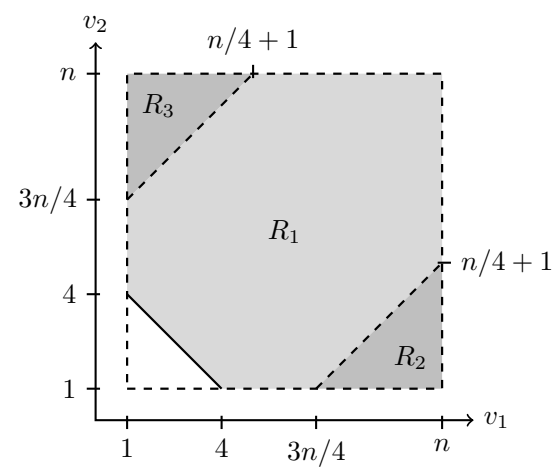

Figure 2: The allocation function for the lottery pricing $\mathcal{L}$.

Note that the distributions are regular. They also have the property that every fixed price yields expected revenue of at most 1 . Thus, as in the previous example, we can think of relaxing the feasibility constraint to allow simultaneous allocation to both reps (or of both items) to get an upper bound of 2 on the expected revenue of any mechanism for the single-parameter setting $\mathcal{I}^{\text {reps }}$ (or for any item pricing for the setting $\mathcal{I}$ ).

The lottery system we consider for this new setting again consists of a price for each item, plus a lottery that gives an equal chance between the items:

$$
\mathcal{L}=\left\{\left(\frac{1}{2}, \frac{1}{2}, \frac{5}{2}\right),\left(1,0,2+\frac{3 H}{8}\right),\left(0,1,2+\frac{3 H}{8}\right)\right\} .
$$

Figure 2 shows the allocation function of this lottery pricing. In particular, $R_{i}$ for $i=1,2,3$ is the set of valuations where lottery $i$ is bought. The probability mass of regions $R_{2}$ and $R_{3}$ together can be computed to be $8 / 3 H-o(1 / H)$. The probability mass of region $R_{1}$ is $2 / 5+(\ln 16) / 25-o(1)$. Therefore, the revenue of $\mathcal{L}$ can be computed to be $2+(\ln 4) / 5-o(1) \approx 2.277-o(1)$. This is a factor of 1.13 higher than the optimal revenue for $\mathcal{I}^{\text {reps }}$, or the revenue of any item pricing for $\mathcal{I}$.

\section{Conclusions and open problems}

In this work, we studied the gap between the revenues of the optimal deterministic and randomized mechanisms for multi-parameter settings with unit-demand agents, and showed that this gap is small when agents' values for different items are independent. Our results extend to a limited form of positive correlation between item values. Several open problems remain. Our bounds on the benefit of randomness are in some cases quite large (although always independent 
of the parameters of the instance) and we believe they can be improved. For example, in the case of a single-agent, we bound the gap by 4 , while the largest gap example known only shows an improvement of approximately 1.13. It would also be interesting to extend our bounds to more general forms of correlation between item values, while avoiding the unbounded gap that Briest et al. (2010) achieve. Another direction of interest is to develop an understanding of special classes of randomized mechanisms, such as those in which the number or the kinds of lotteries offered is restricted.

\section{Acknowledgments}

We thank Jason Hartline and Robert Kleinberg for many helpful discussions.

\section{References}

Aigner, M., 1997. Combinatorial theory. Classics in mathematics, Springer-Verlag.

Alaei, S., 2011. Bayesian combinatorial auctions: Expanding single buyer mechanisms to many buyers, in: Proceedings of the 52nd Annual IEEE Symposium on Foundations of Computer Science, IEEE Computer Society, Washington, DC, USA. pp. 512-521.

Armstrong, M., 1999. Price discrimination by a many-product firm. Review of Economic Studies 66, 151-68.

Balcan, M.F., Blum, A., 2006. Approximation algorithms and online mechanisms for item pricing, in: Proceedings of the 7th ACM Conference on Electronic Commerce, ACM, New York, NY, USA. pp. 29-35.

Balcan, M.F., Blum, A., Mansour, Y., 2008. Item pricing for revenue maximization, in: Proceedings of the 9th ACM Conference on Electronic Commerce, ACM, New York, NY, USA. pp. 50-59.

Briest, P., 2006. Towards Hardness of Envy-Free Pricing. Technical Report TR06-150. Electronic Colloquium on Computational Complexity (ECCC).

Briest, P., Chawla, S., Kleinberg, R., Weinberg, S.M., 2010. Pricing randomized allocations, in: Proceedings of the 21st Annual ACM-SIAM Symposium on Discrete Algorithms, SIAM, Philadelphia, PA, USA. pp. 585-597.

Brualdi, R.A., 1969. Comments on bases in dependence structures. Bulletin of the Australian Mathematical Society $1,161-167$.

Cai, Y., Daskalakis, C., 2011. Extreme-value theorems for optimal multidimensional pricing, in: Proceedings of the 52nd Annual IEEE Symposium on Foundations of Computer Science, IEEE Computer Society, Washington, DC, USA. pp. 522-531.

Cai, Y., Daskalakis, C., Weinberg, S.M., 2012. An algorithmic characterization of multi-dimensional mechanisms, in: Proceedings of the 44th ACM Symposium on Theory of Computing, pp. 459-478.

Chawla, S., Hartline, J., Kleinberg, R., 2007. Algorithmic pricing via virtual valuations, in: Proceedings of the 8th ACM Conference on Electronic Commerce, pp. 243-251.

Chawla, S., Hartline, J.D., Malec, D.L., Sivan, B., 2010. Multi-parameter mechanism design and sequential posted pricing, in: Proceedings of the 42nd ACM Symposium on Theory of Computing, ACM, New York, NY, USA. pp. 311-320.

Daskalakis, C., Weinberg, S.M., 2012. Symmetries and optimal multi-dimensional mechanism design, in: Proceedings of the 13th ACM Conference on Electronic Commerce, pp. 370-387.

Devanur, N., Hartline, J., Karlin, A., Nguyen, T., 2011. Prior-independent multi-parameter mechanism design, in: Chen, N., Elkind, E., Koutsoupias, E. (Eds.), Internet and Network Economics. Springer Berlin / Heidelberg. volume 7090 of Lecture Notes in Computer Science, pp. 122-133. 
Dobzinski, S., Dughmi, S., 2009. On the power of randomization in algorithmic mechanism design, in: Proceedings of the 50th Annual IEEE Symposium on Foundations of Computer Science, IEEE Computer Society, Washington, DC, USA. pp. 505-514.

Dughmi, S., Roughgarden, T., 2010. Black-box randomized reductions in algorithmic mechanism design, in: Proceedings of the 51st Annual IEEE Symposium on Foundations of Computer Science, IEEE Computer Society, Washington, DC, USA. pp. 775-784.

Guruswami, V., Hartline, J.D., Karlin, A.R., Kempe, D., Kenyon, C., McSherry, F., 2005. On profit-maximizing envy-free pricing, in: Proceedings of the 16th Annual ACM-SIAM Symposium on Discrete Algorithms, SIAM, Philadelphia, PA, USA. pp. 1164-1173.

Hartline, J., Karlin, A., 2007. Profit maximization in mechanism design, in: Nisan, N., Roughgarden, T., Tardos, E., Vazirani, V.V. (Eds.), Algorithmic Game Theory. Cambridge Press. chapter 13, pp. 331-362.

Korte, B., Hausmann, D., 1978. An analysis of the greedy heuristic for independence systems, in: B. Alspach, P.H., Miller, D. (Eds.), Algorithmic Aspects of Combinatorics. Elsevier. volume 2 of Annals of Discrete Mathematics, pp. $65-74$.

Manelli, A.M., Vincent, D.R., 2006. Bundling as an optimal selling mechanism for a multiple-good monopolist. Journal of Economic Theory 127, 1-35.

Manelli, A.M., Vincent, D.R., 2007. Multidimensional mechanism design: Revenue maximization and the multiplegood monopoly. Journal of Economic Theory 137, 153-185.

McAfee, R., McMillan, J., 1988. Multidimensional incentive compatibility and mechanism design. Journal of Economic Theory 46, 335-354.

Myerson, R., 1981. Optimal auction design. Mathematics of Operations Research 6, 58-73.

Pavlov, G., 2006. Optimal Mechanism for Selling Substitutes. Boston University - Department of Economics Working Papers Series WP2006-014. Boston University - Department of Economics.

Riley, J., Zeckhauser, R., 1983. Optimal selling strategies: When to haggle, when to hold firm. The Quarterly Journal of Economics 98, 267-289.

Rochet, J.C., Chone, P., 1998. Ironing, Sweeping, and Multidimensional Screening. Econometrica 66, 783-826.

Roughgarden, T., Talgam-Cohen, I., Yan, Q., 2012. Supply-limiting mechanisms, in: Proceedings of the 13th ACM Conference on Electronic Commerce, ACM, New York, NY, USA. pp. 844-861.

Thanassoulis, J.E., 2004. Haggling Over Substitutes. Journal of Economic Theory 117, 217-245.

Yan, Q., 2011. Mechanism design via correlation gap, in: Proceedings of the 22nd Annual ACM-SIAM Symposium on Discrete Algorithms, SIAM, Philadelphia, PA, USA. pp. 710-719. 


\section{Appendix A. Proof of Proposition 10}

Here, we present the proof of Proposition 10. The proof relies on the following well known theorem (Brualdi, 1969).

Theorem 21. If $B_{1}$ and $B_{2}$ are two bases of a matroid, then there exists a one to one function $g: B_{2} \rightarrow B_{1}$ such that $\left(B_{1} \backslash\{g(e)\}\right) \cup\{e\}$ is a basis for all $e \in B_{2}$.

Proof of Proposition 10: In order to apply Theorem 21 we need two bases. Let $B$ be a basis of $\mathcal{S}$. Repeatedly apply the augmentation property to $B_{2}$ and $B$ to produce a basis $\bar{B}_{2} \supset B_{2}$, and then do the same with $B_{1}$ and $\bar{B}_{2}$ to produce a basis $\bar{B}_{1} \supset B_{1}$. Now, Theorem 21 guarantees us a one to one function $g: \bar{B}_{2} \rightarrow \bar{B}_{1}$ such that for all $e \in \bar{B}_{2}$, $\bar{B}_{1} \backslash\{g(e)\} \cup\{e\}$ is independent. Note that for all $e \in \bar{B}_{2} \cap \bar{B}_{1}$, we must have that $g(e)=e$, since otherwise

$$
\bar{B}_{1} \backslash\{g(e)\} \cup\{e\}=\bar{B}_{1} \backslash\{g(e)\} \subsetneq \bar{B}_{1},
$$

and so is not a basis. Since $g$ is one to one, this means that $g(e) \in \overline{B_{1}} \backslash \overline{B_{2}}$ for all $e \in \overline{B_{2}} \backslash \overline{B_{1}}$.

Set $B_{2}{ }^{\prime}=B_{2} \backslash \bar{B}_{1} \subset \bar{B}_{2} \backslash \bar{B}_{1}$. Since $\bar{B}_{1} \backslash \bar{B}_{2} \subset B_{1}$, we may view $g$ as a one to one function $g: B_{2}{ }^{\prime} \rightarrow B_{1}$. It has the first specified property, since for any $e \in B_{2}{ }^{\prime}, B_{1} \backslash\{g(e)\} \cup\{e\} \subset \bar{B}_{1} \backslash\{g(e)\} \cup\{e\}$ is independent. Furthermore, $e \in B_{2} \backslash B_{2}{ }^{\prime} \subset \bar{B}_{1}$ implies $B_{1} \cup\{e\} \subset \bar{B}_{1}$ is independent, and so the second specified property holds as well. 